\title{
The $\nu_{R}$-philic scalar: its loop-induced interactions and Yukawa forces in LIGO observations
}

\author{
Xun-Jie Xu \\ Max-Planck-Institut für Kernphysik, \\ Postfach 103980, D-69029 Heidelberg, Germany \\ E-mail: xunjie.xu@gmail.com
}

ABSTRACT: Right-handed neutrinos $\left(\nu_{R}\right)$ are often considered as a portal to new hidden physics. It is tempting to consider a gauge singlet scalar $(\phi)$ that exclusively couples to $\nu_{R}$ via a $\nu_{R} \nu_{R} \phi$ term. Such a $\nu_{R}$-philic scalar does not interact with charged fermions at tree level but loop-induced effective interactions are inevitable, which are systematically investigated in this work. The magnitude of the loop-induced couplings coincidentally meets the current sensitivity of fifth-force searches. In particular, the loop-induced coupling to muons could be tested in the recent LIGO observations of neutron star mergers as there might be a sizable Yukawa force in the binary system mediated by the $\nu_{R}$-philic scalar.

Keywords: Beyond Standard Model, Neutrino Physics, Solar and Atmospheric Neutrinos ARXIV EPRINT: 2007.01893 


\section{Contents}

1 Introduction 1

2 The model 3

2.1 Notations 3

2.2 Lagrangian 3

2.3 Interactions in the mass basis 5

3 Loop-induced interactions of $\phi$ with charged leptons 5

4 Generalization to three flavors $\quad 9$

$\begin{array}{llr}5 & \text { Phenomenology } & 10\end{array}$

6 Conclusions and discussions 13

$\begin{array}{ll}\text { A Full expressions of } F_{1} \text { and } F_{2} & 14\end{array}$

$\begin{array}{ll}\text { B Some cancellations } & \mathbf{1 5}\end{array}$

$\begin{array}{lll}\text { B.1 Cancellation of UV divergences } & 15\end{array}$

B.2 Cancellation of $\xi$ dependence in $R_{\xi}$ gauges 16

$\begin{array}{lll}\text { B.3 GIM-like cancellation } & 17\end{array}$

$\begin{array}{lr}\text { C Pseudo-scalar couplings } & 19\end{array}$

$\begin{array}{ll}\text { D Generalization to three flavors } & 20\end{array}$

\section{Introduction}

Right-handed neutrinos $\left(\nu_{R}\right)$ are one of the most intriguing pieces to be added to the Standard Model (SM). Not only can they resolve several problems of the SM including neutrinos masses, dark mater, and baryon asymmetry of the universe ${ }^{1}{ }^{1}$ their singlet nature under the SM gauge symmetry also allows for couplings to hidden or dark sectors, a feature known as the neutrino portal to physics beyond the SM.

Among various new physics extensions built on $\nu_{R}$, a gauge singlet scalar $\phi$ coupled exclusively to $\nu_{R}$, referred to as the $\nu_{R}$-philic scalar, is arguably the simplest. ${ }^{2}$ At tree level, the $\nu_{R}$-philic scalar does not interact directly with normal matter that consists of electrons and quarks, which implies that it might have been well hidden from low-energy laboratory

\footnotetext{
${ }^{1}$ See, e.g., the so-called $\nu$ MSM $[1,2]$ which extends the SM by $\nu_{R}$ to incorporate neutrino masses, dark mater, and leptogenesis simultaneously.

${ }^{2}$ It has recently been shown that the $\nu_{R}$-philic scalar could assist low-scale leptogenesis [3].
} 
searches. At the one-loop level, there are loop-induced couplings of $\phi$ to charged fermions, which are suppressed by neutrino masses $\left(m_{\nu}\right)$ in the framework of Type I seesaw [4-8]. The suppression can be understood from that in the zero limit of neutrino masses, which corresponds to vanishing couplings of the SM Higgs to $\nu_{R}$ and left-handed neutrinos $\left(\nu_{L}\right)$, the $\nu_{R}$ sector would be entirely decoupled from the SM content. As we will show, for electrons, the loop-induced effective Yukawa coupling is of the order of

$$
\frac{G_{F} m_{e} m_{\nu}}{16 \pi^{2}} \sim \mathcal{O}\left(10^{-21}\right)
$$

where $G_{F}$ is the Fermi constant and $m_{e}$ is the electron mass.

Despite the small value of the loop-induced coupling, the magnitude coincides with the sensitivity of current precision tests of gravity. For long-range forces mediated by ultralight bosons coupled to electrons or quarks, experimental tests of the strong (based on the lunar laser-ranging technology [9]) and weak (e.g., torsion-balance experiments [10, 11]) equivalence principles are sensitive to Yukawa/gauge couplings spanning from $10^{-20}$ to $10^{-24}$. Very recently, gravitational waves from black hole $(\mathrm{BH})$ and neutron star (NS) binary mergers have been detected by the LIGO/VIRGO collaboration [12, 13], providing novel methods to test theories of gravity as well as other long-range forces [14-24]. For instance, the process of $\mathrm{BH}$ superradiance can be used to exclude a wide range of ultra-light boson masses [15]. The sizable abundance of muons in NS binary systems allows us to probe muonic forces as they could modify the orbital dynamics. It is expected that [24] current and future observations of NS binaries are sensitive to muonic Yukawa/gauge couplings ranging from $10^{-18}$ to $10^{-22}$ which, again, coincidentally covers the theoretical expectation of the loop-induced coupling for muons, $G_{F} m_{\mu} m_{\nu} /\left(16 \pi^{2}\right) \sim 10^{-19}$.

In light of the frontiers of precision and novel tests of gravity and gravity-like forces, it is important to perform an in-depth study on the loop-induced interactions of the $\nu_{R^{-}}$ philic scalar, which is the main goal of this work. We note here that in the seminal work on majorons [25], similar loop-induced interactions have been computed and confronted with experimental limits in the 1980s. More recently, ref. [26] studied majoron decay caused by the loop-induced couplings to charged fermions. In addition, majoron decay to photons is also possible at two-loop level [27]. While the majoron considered in refs. [2527 is a pseudo-scalar boson, in this work we compute loop-induced interactions for a generic scalar and take three lepton flavors into account, with loop calculation details presented. The loop-induced interactions computed in this work could be of importance in phenomenological studies of long-range forces [28-46].

The paper is organized as follows. In section 2, we briefly review the Type I seesaw extended by a gauge singlet scalar, and derive the tree-level interactions for later use. In section 3 , we compute the loop-induced interactions of $\phi$ with charged fermions. The calculation, for simplicity, is first performed assuming only one generation of leptons and then generalized to three flavors in section 4. In section 5, we confront the theoretical predictions to experimental limits including searches for long-range forces of normal matter and the LIGO observations of NS events which are sensitive to muonic couplings. We conclude in section 6 and delegate some details of our calculations to the appendix. 


\section{The model}

\subsection{Notations}

Throughout this paper, Weyl spinors are frequently used in our discussions for simplicity. On the other hand, for Feynman diagram calculations, Dirac or Majorana spinors are more convenient due to a variety of techniques and especially many modern computation packages that have been developed. As both will used in this paper, it is necessary to clarify our notations regarding Weyl spinors versus Dirac/Majorana spinors.

All four-component Dirac/Majorana spinors in this paper are denoted by $\psi_{X}$ with some interpretative subscripts $X$. Otherwise, they are Weyl spinors. For instance, $\nu_{L}$ and $\ell_{R}$ are Weyl spinors of a left-handed neutrino and a right-handed charged lepton, respectively. In contrast to that, $\psi_{\ell}$ is a Dirac spinor of a charged lepton containing both left- and right-handed components.

For Weyl spinors, our notation follows the convention in ref. [47]. For example, the mass and kinetic terms of $\nu_{R}$ are

$$
M_{R} \nu_{R} \nu_{R} \equiv M_{R}\left(\nu_{R}\right)^{\alpha}\left(\nu_{R}\right)_{\alpha}, \quad \nu_{R}^{\dagger} \bar{\sigma}^{\mu} i \partial_{\mu} \nu_{R} \equiv\left(\nu_{R}^{\dagger}\right)_{\dot{\alpha}}\left(\bar{\sigma}^{\mu}\right)^{\dot{\alpha} \beta} i \partial_{\mu}\left(\nu_{R}\right)_{\beta} .
$$

Here and henceforth, the Weyl spinor indices $\alpha, \dot{\alpha}, \beta$ will be suppressed.

Dirac and Majorana spinors can be built from Weyl spinors. Hence the Dirac spinors of charged leptons and neutrinos can be written as

$$
\psi_{\ell}=\left(\begin{array}{c}
\ell_{L} \\
\ell_{R}^{\dagger}
\end{array}\right), \psi_{\nu}=\left(\begin{array}{c}
\nu_{L} \\
\nu_{R}^{\dagger}
\end{array}\right) .
$$

The Majorana spinor of a neutrino mass eigenstate $\nu_{i}$ (where $i=1,2,3, \cdots$ ) is defined as

$$
\psi_{i} \equiv\left(\begin{array}{c}
\nu_{i} \\
\nu_{i}^{\dagger}
\end{array}\right) .
$$

Note that it is self-conjugate: $\psi_{i}^{c}=\psi_{i}$. For later convenience, some identities are listed below to convert Weyl spinors into Dirac/Majorana spinors:

$$
\begin{aligned}
& \nu_{i} \nu_{j}=\nu_{j} \nu_{i}=\overline{\psi_{i}} P_{L} \psi_{j}, \quad \nu_{i}^{\dagger} \nu_{j}^{\dagger}=\nu_{j}^{\dagger} \nu_{i}^{\dagger}=\overline{\psi_{i}} P_{R} \psi_{j}, \quad \nu_{i}^{\dagger} \overline{\sigma^{\mu}} \nu_{j}=\overline{\psi_{i}} \gamma^{\mu} P_{L} \psi_{j}, \\
& \ell_{L} \nu_{i}=\nu_{i} \ell_{L}=\overline{\psi_{i}} P_{L} \psi_{\ell}, \quad \ell_{R} \nu_{i}=\nu_{i} \ell_{R}=\overline{\psi_{\ell}} P_{L} \psi_{i}, \quad \ell_{L}^{\dagger} \bar{\sigma}^{\mu} \nu_{i}=\overline{\psi_{\ell}} \gamma^{\mu} P_{L} \psi_{i} \text {, }
\end{aligned}
$$

where $P_{L / R} \equiv\left(1 \mp \gamma^{5}\right) / 2$ and $\gamma_{L}^{\mu} \equiv \gamma^{\mu} P_{L}$.

\subsection{Lagrangian}

We consider the SM extended by several right-handed neutrinos $\nu_{R}$ and a singlet scalar $\phi$. In Type I seesaw, the number of $\nu_{R}$ needs to be $\geq 2$ in order to accommodate the observed neutrino oscillation data. Let us start with one generation of leptons and ignore the flavor structure (for the realistic case including three generations, see section 4). The Lagrangian of $\nu_{R}$ and $\phi$ reads:

$$
\mathcal{L} \supset \nu_{R}^{\dagger} \bar{\sigma}^{\mu} i \partial_{\mu} \nu_{R}+\frac{1}{2}(\partial \phi)^{2}+\frac{1}{2} m_{\phi}^{2} \phi^{2}+\left[\frac{M_{R}}{2} \nu_{R} \nu_{R}+\frac{y_{R}}{2} \nu_{R} \nu_{R} \phi+\text { h.c. }\right] .
$$


Here we assume $\phi$ is a real scalar or pseudo-scalar field. If it is a complex field, one can decompose it as $\phi=\phi_{r}+i \phi_{i}$ with $\phi_{r}$ and $\phi_{i}$ being real scalar and pseudo-scalar fields respectively. To make our calculation applicable to both scalar and pseudo-scalar cases, we allow $y_{R}$ to be a complex coupling.

The Dirac masses of leptons are generated by

$$
\mathcal{L} \supset y_{\nu} \widetilde{H}^{\dagger} L \nu_{R}+y_{\ell} H^{\dagger} L \ell_{R}+\text { h.c. }
$$

where $H$ is the SM Higgs doublet $\left(\widetilde{H} \equiv i \sigma_{2} H^{*}\right), L=\left(\nu_{L}, \ell_{L}\right)^{T}$ is a left-handed lepton doublet, and $\ell_{R}$ is a right-handed charged lepton. After electroweak symmetry breaking, $\langle H\rangle=(0, v)^{T} / \sqrt{2}$, eq. (2.7) leads to the following mass terms:

$$
\mathcal{L} \supset m_{D} \nu_{L} \nu_{R}+m_{\ell} \ell_{L} \ell_{R}+\text { h.c. },
$$

where

$$
m_{D} \equiv y_{\nu} \frac{v}{\sqrt{2}}, m_{\ell} \equiv y_{\ell} \frac{v}{\sqrt{2}}
$$

The Dirac and Majorana mass terms of neutrinos can be formulated as

$$
\mathcal{L}_{\nu \text { mass }}=\frac{1}{2}\left(\nu_{L}, \nu_{R}\right)\left(\begin{array}{cc}
0 & m_{D} \\
m_{D} & M_{R}
\end{array}\right)\left(\begin{array}{c}
\nu_{L} \\
\nu_{R}
\end{array}\right)
$$

which then can be diagonalized by

$$
\left(\begin{array}{c}
\nu_{L} \\
\nu_{R}
\end{array}\right)=U\left(\begin{array}{l}
\nu_{1} \\
\nu_{4}
\end{array}\right), U^{T}\left(\begin{array}{cc}
0 & m_{D} \\
m_{D} & M_{R}
\end{array}\right) U=\left(\begin{array}{ll}
m_{1} & \\
& m_{4}
\end{array}\right)
$$

Here $\nu_{1}$ and $\nu_{4}$ are the light and heavy mass eigenstates with their masses determined by

$$
m_{1}=\frac{1}{2}\left(\sqrt{4 m_{D}^{2}+M_{R}^{2}}-M_{R}\right), m_{4}=\frac{1}{2}\left(\sqrt{4 m_{D}^{2}+M_{R}^{2}}+M_{R}\right) .
$$

The unitary matrix $U$ is parametrized as

$$
U=\left(\begin{array}{cc}
-i c_{\theta} & s_{\theta} \\
i s_{\theta} & c_{\theta}
\end{array}\right)
$$

where $c_{\theta} \equiv \cos \theta, s_{\theta} \equiv \sin \theta$, and

$$
\theta=\arctan \sqrt{m_{1} / m_{4}}
$$

Eq. (2.13) has been parametrized in such a way that $m_{D}, M_{R}, m_{1}$ and $m_{4}$ are all positive numbers. 


\subsection{Interactions in the mass basis}

Since $\nu_{L}$ and $\nu_{R}$ are not mass eigenstates, we need to reformulate neutrino interactions in the mass basis, i.e., the basis of $\nu_{1}$ and $\nu_{4}$. The two bases are related by

$$
\begin{aligned}
& \nu_{L}=-i c_{\theta} \nu_{1}+s_{\theta} \nu_{4}, \\
& \nu_{R}=i s_{\theta} \nu_{1}+c_{\theta} \nu_{4} .
\end{aligned}
$$

Neutrino interactions in the original basis (chiral basis) include gauge interactions and Yukawa interactions, summarized as follows:

$$
\mathcal{L} \supset \frac{g}{2 c_{W}} Z_{\mu} \nu_{L}^{\dagger} \bar{\sigma}^{\mu} \nu_{L}+\left[\frac{g}{\sqrt{2}} W_{\mu}^{-} \ell_{L}^{\dagger} \bar{\sigma}^{\mu} \nu_{L}-y_{\nu} H^{+} \ell_{L} \nu_{R}+y_{\ell} H^{-} \nu_{L} \ell_{R}+\frac{y_{R}}{2} \nu_{R} \nu_{R} \phi+\text { h.c. }\right]
$$

where $g$ is the gauge coupling of $\mathrm{SU}(2)_{L}$ in the $\mathrm{SM}, c_{W}$ is the cosine of the Weinberg angle, and $H^{ \pm}$is the charged component of $H$, i.e. the Goldstone boson associated to $W^{ \pm}$.

Now applying the basis transformation in eqs. (2.15) and (2.16) to eq. (2.17), we get

$$
\mathcal{L} \supset g_{Z}^{i j} Z_{\mu} \nu_{i}^{\dagger} \bar{\sigma}^{\mu} \nu_{j}+\left[g_{W}^{i} W_{\mu}^{-} \ell_{L}^{\dagger} \bar{\sigma}^{\mu} \nu_{i}-y_{\nu}^{i} H^{+} \ell_{L} \nu_{i}+y_{\ell}^{i} H^{-} \nu_{i} \ell_{R}+\frac{y_{R}^{i j}}{2} \nu_{i} \nu_{j} \phi+\text { h.c. }\right] \text {. }
$$

Here $i$ and $j$ take either 1 or 4 . The couplings $g_{Z}^{i j}, g_{W}^{i}, y_{\nu}^{i}, y_{\ell}^{i}, y_{R}^{i j}$ are given by the following matrices or vectors:

$$
\begin{aligned}
& g_{Z}^{i j}=\frac{g}{2 c_{W}}\left(\begin{array}{cc}
c_{\theta}^{2} & i c_{\theta} s_{\theta} \\
-i c_{\theta} s_{\theta} & s_{\theta}^{2}
\end{array}\right), \quad y_{R}^{i j}=y_{R}\left(\begin{array}{cc}
-s_{\theta}^{2} & i c_{\theta} s_{\theta} \\
i c_{\theta} s_{\theta} & c_{\theta}^{2}
\end{array}\right), \\
& g_{W}^{i}=\frac{g}{\sqrt{2}}\left(-i c_{\theta}, s_{\theta}\right), \quad y_{\nu}^{i}=y_{\nu}\left(i s_{\theta}, c_{\theta}\right), \quad y_{\ell}^{i}=y_{\ell}\left(-i c_{\theta}, s_{\theta}\right) \text {. }
\end{aligned}
$$

Eq. (2.18) can be straightforwardly expressed in terms of Dirac and Majorana spinors according to eqs. (2.4) and (2.5):

$$
\mathcal{L} \supset g_{Z}^{i j} Z_{\mu} \overline{\psi_{i}} \gamma_{L}^{\mu} \psi_{j}+\left[g_{W}^{i} W_{\mu}^{-} \overline{\psi_{\ell}} \gamma_{L}^{\mu} \psi_{i}+H^{-} \overline{\psi_{\ell}}\left(y_{\ell}^{i} P_{L}-y_{\nu}^{i *} P_{R}\right) \psi_{i}+\frac{y_{R}^{i j}}{2} \overline{\psi_{i}} P_{L} \psi_{j} \phi+\text { h.c. }\right] \text {. }
$$

Note that in the mass basis, $\phi$ couples to both heavy and light neutrinos but the coupling of the latter is suppressed by $s_{\theta}$.

\section{Loop-induced interactions of $\phi$ with charged leptons}

As shown in the previous section, at tree level the scalar singlet $\phi$ only couples to neutrinos, including light and heavy ones in the mass basis. It does not interact with other fermions directly. In this section, we show that one-loop corrections lead to effective interactions of $\phi$ with charged leptons.

From eq. (2.18), it is straightforward to check that at the one-loop level, in the unitarity gauge (which means Goldstone boson interactions can be ignored), there are only two 

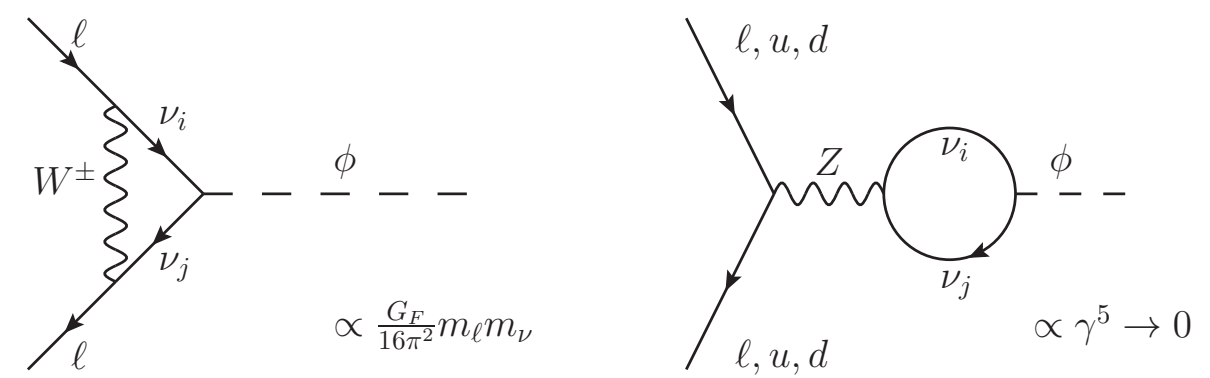

Figure 1. One-loop diagrams that give rise to effective couplings of $\phi$ with charged leptons $(\ell)$ or quarks $(u, d)$. The left diagram is computed in eqs. (3.10)-(A.2), and the right diagram leads to a pseudo-scalar coupling (with $\gamma^{5}$ ), the effect of which however is suppressed in unpolarized matter. The diagrams are presented in the mass basis $\left(\nu_{i}\right.$ and $\nu_{j}$ are mass eigenstates). For an equivalent description in the chiral basis, see figure 2 .

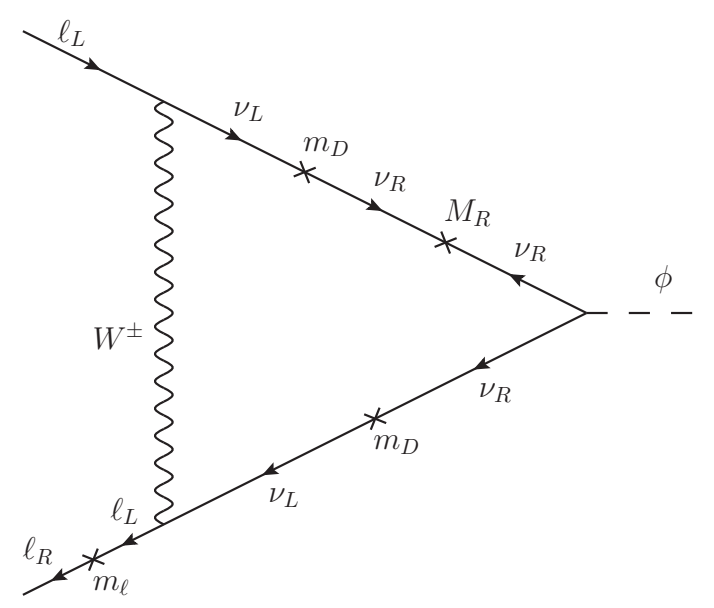

Figure 2. The $W^{ \pm}$-mediated loop diagram in the chiral basis, which is equivalent to the left diagram in figure 1 in the mass basis. It shows explicitly how chirality changes in the process. Since in the chiral basis $W^{ \pm}$only couples to left-handed leptons and $\phi$ only to $\nu_{R}$, we need two mass insertions of $m_{D}$ to connect $\nu_{L}$ and $\nu_{R}$. Other two mass insertions, $M_{R}$ and $m_{\ell}$, are also necessary due to additional requirements — see discussions in the text.

possible diagrams that can connect $\phi$ to charged leptons or quarks, as shown in figure 1. The second diagram involving the $Z$ boson actually leads to a pseudo-scalar coupling (see calculations later on). In unpolarized matter, pseudo-scalar interactions cannot cause significant long-range forces $[48,49]$ because the Yukawa potential between two fermions are spin dependent. When taking an average over the spins, the effect of pseudo-scalar interactions vanishes. Therefore, we will focus our discussions on the first diagram where the external fermion lines have to be charged leptons.

The diagrams in figure 1 are in the mass basis which is technically convenient for evaluation. Nonetheless it is illuminating to show figure 2, another diagram in the chiral basis which explicitly shows how chirality changes in the process. The physical results should be basis independent. 
Figure 2 follows directly from eq. (2.17), which suggests that $\phi$ only couples to $\nu_{R}$ while $W^{ \pm}$interacts with $\nu_{L}$. Therefore, two Dirac mass insertions $\left(m_{D} \nu_{L} \nu_{R}\right.$ and $\left.m_{D} \nu_{L}^{\dagger} \nu_{R}^{\dagger}\right)$ are necessarily introduced to connect $\nu_{R}$ and $\nu_{L}$, or $\nu_{R}^{\dagger}$ and $\nu_{L}^{\dagger}$. Note that the two $W^{ \pm}$vertices have to be conjugate to each other, which implies that from the $W^{ \pm}$side, a pair of $\nu_{L}$ and $\nu_{L}^{\dagger}$ is provided. On the other hand, the Yukawa vertex couples $\phi$ to two $\nu_{R}$ 's rather than a pair of $\nu_{R}$ and $\nu_{R}^{\dagger}$. So a Majorana mass insertion is required to flip the lepton number and convert one of them to $\nu_{R}^{\dagger}$. The direction of lepton-number flow in this diagram are represented by the arrows. Note that according to the conventions in section 2.1, $\nu_{L}$ and $\nu_{R}$ have opposite lepton numbers. So for $\nu_{R} \nu_{R} \phi$, the arrow of $\nu_{R}$ should be outgoing. In contrast to that, the arrow of $\nu_{L}$ in the $W_{\mu}^{-} \ell_{L}^{\dagger} \bar{\sigma}^{\mu} \nu_{L}$ vertex goes inwardly. Finally, there should be a mass insertion of $m_{\ell} \ell_{L} \ell_{R}$ on one of the external fermion lines because it is impossible to write down an effective operator that consists of $\phi$ and two $\ell_{L}$ 's - the operator $\phi \ell_{L} \ell_{L}$ is not allowed due to electric charge conservation.

The chirality analysis in figure 2 indicates that the diagram would be proportional to $m_{D}{ }^{2} M_{R} m_{\ell}$ if all these masses are sufficiently small. If $M_{R}$ is much larger than the typical scale of the loop momentum, then the propagators of $\nu_{R}$ also contribute an additional factor of $M_{R}^{-2}$. In this case, the diagram is expected to be proportional to $m_{D}^{2} M_{R}^{-1} m_{\ell} \sim m_{\nu} m_{\ell}$ where $m_{\nu}$ is the light neutrino mass.

Now let us compute the loop diagrams explicitly. Using the Dirac/Majorana spinor representation in eq. (2.21), we can write down the amplitudes of the two diagrams in figure 1:

$$
\begin{aligned}
i \mathcal{M}_{W} & =(i)^{3} \int \frac{d^{4} k}{(2 \pi)^{4}} \overline{u\left(p_{2}\right)} g_{W}^{j} \gamma_{L}^{\mu} \Delta_{j}\left(p_{j}\right) \frac{y_{R}^{j i} P_{L}+y_{R}^{j i *} P_{R}}{2} \Delta_{i}\left(p_{i}\right) g_{W}^{i *} \gamma_{L}^{\nu} u\left(p_{1}\right) \Delta_{\mu \nu}^{W}(k), \\
i \mathcal{M}_{Z} & =(i)^{3} \int \frac{d^{4} p_{i}}{(2 \pi)^{4}} \overline{u\left(p_{2}\right)} g_{Z}^{(\ell)} \gamma_{L}^{\mu} u\left(p_{1}\right) \operatorname{tr}\left[-g_{Z}^{i j} \gamma_{L}^{\nu} \Delta_{j}\left(p_{j}\right) \frac{y_{R}^{j i} P_{L}+y_{R}^{j i *} P_{R}}{2} \Delta_{i}\left(p_{i}\right)\right] \Delta_{\mu \nu}^{Z}(q),
\end{aligned}
$$

where $(i)^{3}$ comes from three vertices; $p_{1}$ and $p_{2}$ are the momenta of the upper and lower external fermion lines; $p_{i}$ and $p_{j}$ are the momenta of $\nu_{i}$ and $\nu_{j} ; q=p_{2}-p_{1}=p_{j}-p_{i}$; $k$ is the momentum of $W$ propagator; and $g_{Z}^{(\ell)}$ is the gauge coupling of $Z$ to the charge fermion $\ell$. The symbol $\Delta$ denotes propagators. For Majorana spinors in the mass basis, their propagators have the same form as Dirac propagators:

$$
\Delta_{i}(p)=\frac{i}{\not p-m_{i}} \text {. }
$$

The propagators of $W^{ \pm}$and $Z$ are gauge dependent. Most generally, in $R_{\xi}$ gauges, they are:

$$
\begin{aligned}
& \Delta_{\mu \nu}^{W}(k)=\frac{-i}{k^{2}-m_{W}^{2}}\left[g_{\mu \nu}-\frac{k_{\mu} k_{\nu}}{k^{2}-\xi m_{W}^{2}}(1-\xi)\right], \\
& \Delta_{\mu \nu}^{Z}(k)=\frac{-i}{k^{2}-m_{Z}^{2}}\left[g_{\mu \nu}-\frac{k_{\mu} k_{\nu}}{k^{2}-\xi m_{Z}^{2}}(1-\xi)\right] .
\end{aligned}
$$

The unitarity gauge corresponds to $\xi \rightarrow \infty$. Except for the unitarity gauge, other gauges with finite $\xi$, e.g., the Feynman-'t Hooft gauge $(\xi=1)$ and the Lorentz gauge $(\xi=0)$, 
require the inclusion of Goldstone boson diagrams. The unitarity gauge, albeit involving fewer diagrams by virtue of infinitely large masses of Goldstone boson propagators, has a disadvantage in that the cancellation of UV divergences is less obvious - see discussions in section B.1. Nonetheless, it is straightforward to compute $i \mathcal{M}_{W}$ and $i \mathcal{M}_{Z}$ for general values of $\xi$.

First, let us inspect the $i \mathcal{M}_{Z}$ amplitude. The loop integral of the trace part gives rise to a quantity proportional to $q^{\nu}$ :

$$
\int \frac{d^{4} p_{i}}{(2 \pi)^{4}} \operatorname{tr}\left[\gamma_{L}^{\nu} \Delta_{j}\left(p_{j}\right) P_{L / R} \Delta_{i}\left(p_{i}\right)\right] \propto q^{\nu}
$$

which can be expected from Lorentz invariance, explained as follows. On the left-hand side of eq. (3.6) there are only two independent momenta $p_{j}=p_{i}+q$ and $p_{i}$. After $p_{i}$ being integrated out, the only quantity that can carry a Lorentz index is $q$ so the result is proportional to $q^{\nu}$. Now plugging this in eq. (3.2), we can immediately get a $\gamma^{5}$ sandwiched between $\overline{u\left(p_{2}\right)}$ and $u\left(p_{1}\right)$ :

$$
\begin{aligned}
\overline{u\left(p_{2}\right)} \not 1 P_{L} u\left(p_{1}\right) & =\overline{u\left(p_{2}\right)}\left(\not p_{2} P_{L}-P_{R} \not p_{1}\right) u\left(p_{1}\right) \\
& =m_{\ell} \overline{u\left(p_{2}\right)}\left(P_{L}-P_{R}\right) u\left(p_{1}\right) \\
& =-m_{\ell} \overline{u\left(p_{2}\right)} \gamma^{5} u\left(p_{1}\right) .
\end{aligned}
$$

Therefore, the $Z$-mediated diagram induces a pseudo-scalar coupling, which is computed in appendix $\mathrm{C}$.

The $i \mathcal{M}_{W}$ amplitude can be computed by splitting the $W^{ \pm}$propagator in eq. (3.4) to two parts:

$$
\Delta_{\mu \nu}^{W}(k)=-i \frac{g_{\mu \nu}-k_{\mu} k_{\nu} / m_{W}^{2}}{k^{2}-m_{W}^{2}}-i \frac{k_{\mu} k_{\nu} / m_{W}^{2}}{k^{2}-\xi m_{W}^{2}},
$$

where the first part does not contain $\xi$ and the second part is important for cancellation of UV divergences. Note that when computing eq. (3.1), because of the chiral projectors in $y_{R}^{j i} P_{L}+y_{R}^{j i *} P_{R}$, the product of Dirac matrices gives

$$
\gamma_{L}^{\mu} \frac{\not p_{j}+m_{j}}{p_{j}^{2}-m_{j}^{2}}\left[y_{R}^{j i} P_{L}+y_{R}^{j i *} P_{R}\right] \frac{\not p_{i}+m_{i}}{p_{i}^{2}-m_{i}^{2}} \gamma_{L}^{\nu}=\gamma_{L}^{\mu} \frac{\not p_{j} m_{i} y_{R}^{j i *}+y_{R}^{j i} m_{j} \not p_{i}}{\left(p_{j}^{2}-m_{j}^{2}\right)\left(p_{i}^{2}-m_{i}^{2}\right)} \gamma_{L}^{\nu} .
$$

It implies that if $m_{i} \rightarrow 0$ and $m_{j} \rightarrow 0$, the result would be zero, which agrees with our analysis in the chiral basis.

With the above details being noted, we compute ${ }^{3}$ eq. (3.1) in the soft scattering limit $(q \rightarrow 0)$ with the approximation of $m_{\ell} \ll m_{W}$ and obtain:

$$
i \mathcal{M}_{W}=i \frac{m_{\ell} G^{i j}}{256 \pi^{2} m_{W}^{2}}\left[F_{1}\left(m_{i}, m_{j}\right)+F_{2}\left(m_{i}, m_{j}\right)\right] \overline{u\left(p_{2}\right)} u\left(p_{1}\right)+i \lambda_{\phi \ell \ell}^{(W)} \overline{u\left(p_{2}\right)} i \gamma^{5} u\left(p_{1}\right)
$$

where

$$
G^{i j} \equiv g_{W}^{i *} g_{W}^{j}\left(m_{j} y_{R}^{i j}+m_{i} y_{R}^{i j *}\right)=\frac{g^{2} c_{\theta}^{2} s_{\theta}^{2}}{2}\left[\begin{array}{cc}
-m_{1}\left(y_{R}+y_{R}^{*}\right) & m_{1} y_{R}^{*}-m_{4} y_{R} \\
m_{1} y_{R}-m_{4} y_{R}^{*} & m_{4}\left(y_{R}+y_{R}^{*}\right)
\end{array}\right],
$$

\footnotetext{
${ }^{3}$ We use Package-X [50] to compute loop integrals analytically and our code is available from [https://github.com/xunjiexu/vR_loop].
} 
and $F_{1}$ and $F_{2}$ correspond to the contributions of the first and second parts of the $W^{ \pm}$ propagator in eq. (3.8), respectively. Their explicit forms are given in appendix A. The second term of eq. (3.10) leads to pseudo-scalar couplings which cannot cause significant effect in unpolarized matter. Nevertheless, we compute the loop-induced pseudo-scalar couplings in appendix C.

We need to sum over $i$ and $j$ in eq. (3.10) to get a finite and gauge independent result. There are several cancellations involved in the summation, which are discussed in detail in appendix B. After a careful treatment of these cancellations, we obtain:

$$
i \mathcal{M}_{W} \approx i \overline{u\left(p_{2}\right)} y_{\phi \ell \ell} u\left(p_{1}\right),
$$

with

$$
y_{\phi \ell \ell}=-\frac{3 G_{F} m_{1} m_{\ell} \operatorname{Re}\left(y_{R}\right)}{16 \sqrt{2} \pi^{2}} .
$$

It implies that the loop diagram generates the effective interaction

$$
\mathcal{L} \supset y_{\phi \ell \ell} \phi \overline{\psi_{\ell}} \psi_{\ell}
$$

where the effective coupling $y_{\phi \ell \ell}$, given in eq. (3.13), is suppressed by the neutrino mass $m_{\nu}$ and the charged lepton mass $m_{\ell}$.

\section{Generalization to three flavors}

So far we have only considered leptons of a single flavor for which we have computed the loop-induced coupling $y_{\phi \ell \ell}$, as given in eq. (3.13). Now we would like to generalize it to the realistic scenario with three flavors.

Assuming there are three generations of $\nu_{L}$ and $\nu_{R}$, we can express the neutrino mass terms in a similar way to eq. (2.10) except that now the mass matrix is interpreted as a $6 \times 6$ matrix:

$$
M_{6 \nu}=\left[\begin{array}{cc}
0 & m_{D} \\
m_{D}^{T} & M_{R}
\end{array}\right]_{6 \times 6},
$$

where $m_{D}$ and $M_{R}$ are $3 \times 3$ Dirac and Majorana mass matrices respectively. In principle, the number of right-handed neutrinos does not have to be three. It can be two or more. But to make it concrete, let us concentrate on the case with three $\nu_{L}$ plus three $\nu_{R}$.

The neutrino mass terms and Yukawa terms are formulated as:

$$
\mathcal{L} \supset \frac{1}{2}\left(\nu_{L}^{T}, \nu_{R}^{T}\right) M_{6 \nu}\left(\begin{array}{c}
\nu_{L} \\
\nu_{R}
\end{array}\right)+\frac{1}{2} \phi \nu_{R}^{T} Y_{R}^{0} \nu_{R}+\text { h.c. },
$$

where $Y_{R}^{0}$ is a $3 \times 3$ Yukawa coupling matrix.

A detailed analysis of this scenario is delegated to appendix D. Here we simply summarize the results. In general, without any requirements of $m_{D}, M_{R}$ and $Y_{R}^{0}$, the loop-induced coupling can be numerically obtained from

$$
y_{\phi \ell \ell}=\frac{G_{F} m_{\ell}}{64 \sqrt{2} \pi^{2}} \sum_{i, j} U_{\ell i}^{*} U_{\ell j}\left(Y_{R} M_{d}+M_{d} Y_{R}^{\dagger}\right)_{i j} F_{12}\left(m_{i}, m_{j}\right),
$$


where $F_{12}$ can be computed using eq. (B.11), $U$ is the full $6 \times 6$ mixing matrix that can diagonalize $M_{6 \nu}, M_{d} \equiv U^{T} M_{6 \nu} U=\operatorname{diag}\left(m_{1}, m_{2}, \cdots, m_{6}\right)$ is the diagonalized form of $M_{6 \nu}$, and $Y_{R}$ is the mass-basis form of $Y_{R}^{0}$ :

$$
Y_{R} \equiv U^{T} \operatorname{diag}\left(0_{3 \times 3}, Y_{R}^{0}\right) U
$$

If $M_{R}$ and $Y_{R}^{0}$ can be simultaneously editorialized, ${ }^{4}$ then without loss of generality, we can assume $M_{R}$ and $Y_{R}^{0}$ are diagonal. Under this assumption, the result can be further simplified to

$$
y_{\phi \ell \ell} \approx-\frac{3 G_{F} m_{\ell}}{32 \sqrt{2} \pi^{2}}\left[m_{D}\left(Y_{R}^{0}+Y_{R}^{0 \dagger}\right) M_{R}^{-1} m_{D}^{\dagger}\right]_{\ell \ell} .
$$

Eq. (4.5) can also be expressed in the Casas-Ibarra parametrization [54]:

$$
y_{\phi \ell \ell} \approx-\frac{3 G_{F} m_{\ell}}{32 \sqrt{2} \pi^{2}}\left[U_{L}^{*} \sqrt{m_{\nu}^{d}} R^{T}\left(Y_{R}^{0}+Y_{R}^{0 \dagger}\right) R^{*} \sqrt{m_{\nu}^{d}} U_{L}^{T}\right]_{\ell \ell}
$$

where $U_{L}$ is the PMNS matrix, $m_{\nu}^{d}=\operatorname{diag}\left(m_{1}, m_{2}, m_{3}\right)$, and $R$ is a complex orthogonal matrix $\left(R R^{T}=1\right)$, which is determined by $m_{D}=i U_{L}^{*} \sqrt{m_{\nu}^{d}} R^{T} \sqrt{M_{R}^{-1}}$ in the Casas-Ibarra parametrization. Note that our convention of $U_{L}$ is chosen in the way that $U_{L}^{T} m_{\nu} U_{L}=m_{\nu}^{d}$ for $m_{\nu} \equiv-m_{D} M_{R}^{-1} m_{D}^{T}$.

\section{Phenomenology}

The loop-induced interaction of $\phi$ with electrons leads to a Yukawa potential between two objects containing $N_{1}$ and $N_{2}$ electrons,

$$
V(r)=-\frac{y_{\phi e e}^{2} N_{1} N_{2}}{4 \pi r} e^{-m_{\phi} r}
$$

The effective Yukawa coupling $y_{\phi e e}$ is of order $G_{F} m_{e} m_{\nu} /\left(16 \pi^{2}\right) \sim \mathcal{O}\left(10^{-21}\right)$, which reaches the current sensitivity of long-range force searches. If we replace electrons with muons, the effective coupling is generally two orders of magnitude larger because $m_{\mu} / m_{e} \approx 200$. The muonic long-range force can be tested in binary systems of neutron stars (NS) which contain $\mathcal{O}(0.1 \sim 1 \%)$ muons of the total mass [55]. In particular, the recent gravitational wave observation of NS binary mergers by the LIGO collaboration $[12,13]$ are able to test the muonic force with unprecedented sensitivity.

As indicated by eq. (4.3), the value of $y_{\phi \ell \ell}$ depends on neutrino masses and the Yukawa couplings of $\phi$ to $\nu_{R}$. Since there are many free parameters in $Y_{R}$ and $M_{\nu}$ (where Majorana phases, the Dirac CP phase, the lightest neutrino mass are still unknown), we would like to simply parametrize $y_{\phi \ell \ell}$ as follows:

$$
\begin{aligned}
y_{\phi e e} & =\frac{3 G_{F} m_{e} Y_{R}^{(e)} m_{\nu}^{(e)}}{16 \sqrt{2} \pi^{2}} \approx 8.0 \times 10^{-22} Y_{R}^{(e)}\left(\frac{m_{\nu}^{(e)}}{0.01 \mathrm{eV}}\right), \\
y_{\phi \mu \mu} & =\frac{3 G_{F} m_{\mu} Y_{R}^{(\mu)} m_{\nu}^{(\mu)}}{16 \sqrt{2} \pi^{2}} \approx 5.0 \times 10^{-19} Y_{R}^{(\mu)}\left(\frac{m_{\nu}^{(\mu)}}{0.03 \mathrm{eV}}\right),
\end{aligned}
$$

\footnotetext{
${ }^{4}$ Such a feature could arise from flavor symmetries, see models in refs. [51-53].
} 


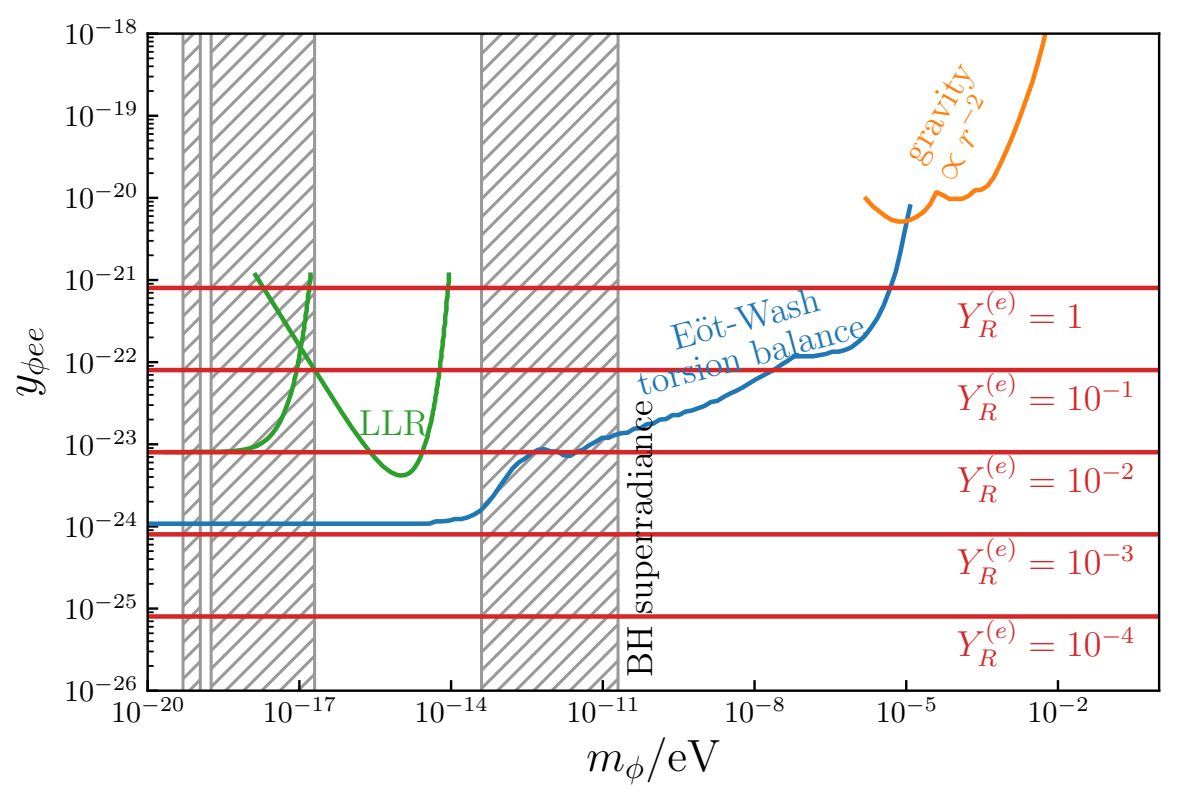

Figure 3. The effective Yukawa coupling of $\phi$ to $e$, compared with experimental limits. The predictions of our model (red) are evaluated according to eq. (5.2) with $m_{\nu}^{(e)}=0.01 \mathrm{eV}$. The experimental limits come from the Eöt-Wash torsion-balance tests of the equivalence principle (blue) [56], tests of gravitational inverse-square law (orange) [57], lunar laser-ranging (LLR, green) measurements $[9,56]$, and black hole superradiance (hatched bands) [15].

where $Y_{R}^{(e)}$ and $Y_{R}^{(\mu)}$ account for the suppression caused by the original Yukawa couplings if they are not of $\mathcal{O}(1)$, while $m_{\nu}^{(e)}$ and $m_{\nu}^{(\mu)}$ account for the suppression due to neutrino masses. In the limit of $Y_{R 1}=Y_{R 2}=Y_{R 3}$ and $U_{\mathrm{PMNS}}^{*}=U_{\mathrm{PMNS}}, m_{\nu}^{(e)}$ would be identical to the neutrino mass matrix element responsible for neutrinoless double beta decay (often denoted as $m_{e e}$ in the literature). But in general, they are different. Since $Y_{R}^{(e)} m_{\nu}^{(e)}$ and $Y_{R}^{(\mu)} m_{\nu}^{(\mu)}$ depend on a lot of unknown fundamental parameters, it is possible that the Majorana phases and other free parameters conspire in such a way that $Y_{R}^{(e)} m_{\nu}^{(e)}=0$ while $Y_{R}^{(\mu)} m_{\nu}^{(\mu)}$ is not suppressed or vice versa, analogous to the well-known fact that $m_{e e}$ for neutrinoless double beta decay can vanish in the normal mass ordering.

Next, we shall confront the theoretical predictions with experimental limits, as shown in figures 3 and 4 for $y_{\phi e e}$ and $y_{\phi \mu \mu}$ respectively.

For $y_{\phi e e}$, current limits come from long-range force searches of normal matter, which have long been investigated in precision tests of gravity, in particular, in tests of the equivalence principle. The Yukawa force mediated by $\phi$ can affect the former by contributing an exponential term to the total force and affect the latter due to its leptophilic coupling, which causes differential free-fall accelerations for different materials. So far, the Eöt-Wash torsion-balance experiment has performed tests of the weak equivalence principle with the highest precision $[10,11]$, leading to the most stringent constraint on $y_{\text {dee }}$ in the regime of very small $m_{\phi}$. In addition, the lunar laser-ranging (LLR) technology which is able to measure the varying distance between the moon and the earth to high precision using laser 


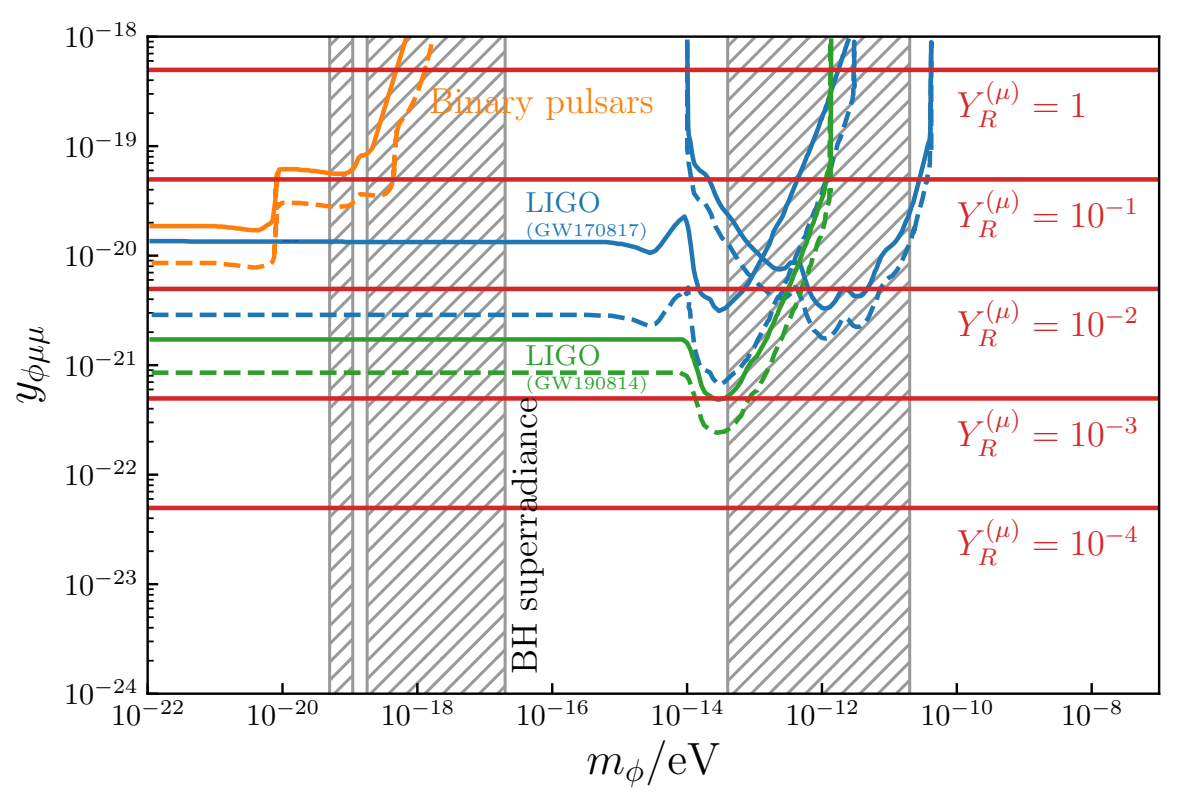

Figure 4. The effective Yukawa coupling of $\phi$ to $\mu$, compared with experimental limits. The predictions of our model (red) are evaluated according to eq. (5.3) with $m_{\nu}^{(\mu)}=0.03 \mathrm{eV}$. The muonic force could be probed in binary systems of neutron stars (NS) due to the considerable abundance of muons. The blue and green curves represent current sensitivity of the LIGO observations of GW170817 (NS-NS merger) and GW190814 (NS-BH merger) events, respectively. Solid (dashed) curves take conservative (optimistic) estimates of the muon abundance [24]. In addition, precision measurements of binary pulsar systems are also sensitive to the muonic force (orange curves) [24].

pulses is also sensitive to new long-range forces [9]. These two bounds, reviewed in ref. [56], are presented in figure 3 and overlap with the theoretically most favored region (red lines).

For larger masses, $y_{\phi e e}$ is constrained by tests of the inverse-square law of gravity $[57,58]$, the Casimir effect [59], stellar cooling processes [60,61], $N_{\text {eff }}$ in cosmology [62-66], supernovae [67-73], neutrino scattering [74-79], etc. But all these bounds are significantly higher than the largest expected values of $y_{\phi e e}$ - see ref. [37] for a recent compilation of these bounds.

In figure 3 (also figure 4), we add hatched bands to represent the constraint from black hole superradiance [15], which is independent of the Yukawa couplings because the effect is caused by $\phi$ coupling to the spacetime.

For $y_{\phi \mu \mu}$, the aforementioned laboratory constraints do not apply since normal matter does not contain muons. Neutron stars, however, can be a powerful probe of muonic forces due to a significant abundance muons in them, which is expected when the Fermi energy exceeds the muon mass. According to the calculations in refs. [24, 55], the number density of muons is typically of $\mathcal{O}(1 \sim 10) \%$ of the total number density, which is lower than but still comparable to the electron number density. ${ }^{5}$

\footnotetext{
${ }^{5}$ See figure 23 in ref. [55] and figure 3 in ref. [24]. In the former, the number densities of protons and electrons are presented. Assuming charge neutrality of the NS, the difference between proton and electron number densities is approximately the muon number density. The latter needs to be converted from mass ratios to number density ratios by multiplying a factor of $m_{\mu} / m_{n}$ where $m_{n}$ is the neutron mass.
} 
In fact, since the electron and muon number densities are of the same order of magnitude while $y_{\phi \ell \ell} \propto m_{\ell}$, for NS binaries we have

$$
F^{(\mu)} \sim\left(\frac{m_{\mu}}{m_{e}}\right)^{2} F^{(e)} \gg F^{(e)}
$$

where $F^{(\mu)}$ and $F^{(e)}$ are the forces caused by muons and electrons respectively.

The recent observations of NS-NS and NS-BH mergers by the LIGO collaboration provide very promising data to probe the muonic force in this model. For a NS-NS merger, the effect of $\phi$ is two-fold [19]. First, the attractive force affects the orbital dynamics in a classical way, i.e., modifying the Kepler's law when $r \sim m_{\phi}^{-1}$. Second, since $\phi$ is a ultralight boson, there is radiation of $\phi$ due to the rotating dipole, which causes extra energy loss. For a NS-BH merger, only the effect of $\phi$ radiation is relevant. An in-depth analysis of the sensitivity to muonic forces based on the recent two events GW170817 (NS-NS merger) and GW190814 (NS-BH merger) has been performed in ref. [24]. Their results have been incorporated in figure 4, where solid (dashed) curves are derived using a conservative (optimistic) estimate of the muon abundance. For GW170817, the sensitivity curves of the two effects are evaluated and presented separately. The first effect (orbital dynamics) are more sensitive than the second to $m_{\phi}$ when it is in the large-mass $\left(10^{-12} \sim 10^{-10} \mathrm{eV}\right)$ regime. In addition to binary mergers, precision measurements of binary pulsars can also be sensitive to muonic forces $[24,80]$.

As shown in figure 4, the LIGO curves cross the red lines of $Y_{R}^{(\mu)}=10^{-3} \sim 1$, which implies that the loop-induced muonic force in this model could be probed in the theoretically most favored regime. Future experiments such as the Einstein Telescope ${ }^{6}$ and Cosmic Explorer [81] can substantially improve the sensitivity to muonic forces and thus have great potential of probing this scenario.

\section{Conclusions and discussions}

The $\nu_{R}$-philic scalar model naturally gives rise to extremely small couplings of charged leptons to a long-range force mediator via loop-level processes. The small values of the loopinduced couplings coincidentally meet the current sensitivity of long-range force searches in laboratories and in astrophysical observations such as the recent detection of GW from NS mergers by LIGO, as we have shown in figures 3 and 4 .

In this model, loop-induced couplings to quarks also exist, due to the $Z$-mediated diagram in figure 1 . However, our calculation shows that only pseudo-scalar couplings are generated in this case, the effect of which is suppressed in unpolarized matter.

Our loop calculation result for the most general three-flavor case is given by eq. (4.3) which, though involving diagonalization of the full $6 \times 6$ mass matrix, can be numerically evaluated. For the special case where $M_{R}$ and $Y_{R}^{0}$ can be simultaneously diagonalized, the result can be further simplified to eq. (4.6), where the dependence on the PMNS matrix is manifestly extracted.

\footnotetext{
${ }^{6}$ See the ET conceptual design document: https://tds.virgo-gw.eu/?call_file=ET-0106C-10.pdf.
} 
Our results can also be used to obtain loop-induced interactions for other similar models that contain the diagrams in figure 1, via proper replacements of the couplings in vertices and masses in propagators. However, one caveat should be noted here that incomplete models where the tree-level couplings of $\phi$ to light neutrino mass eigenstates are not governed by the active-sterile neutrino mixing would lead to gauge dependent results.

\section{Acknowledgments}

We thank Andreas Trautner and Toby Opferkuch for useful discussions.

\section{A Full expressions of $F_{1}$ and $F_{2}$}

The explicit expressions of $F_{1}$ and $F_{2}$ read as follows:

$$
\begin{aligned}
& F_{1}=6\left(\frac{1}{\epsilon}+\log \frac{\mu^{2}}{m_{W}^{2}}\right)-2 \frac{m_{j}^{2}+2 m_{W}^{2}}{m_{j}^{2}-m_{W}^{2}} \log \frac{m_{j}^{2}}{m_{W}^{2}} \\
& +\frac{5 m_{i}^{2} m_{j}^{2}-5 m_{i}^{2} m_{W}^{2}-5 m_{j}^{2} m_{W}^{2}+11 m_{W}^{4}}{\left(m_{i}^{2}-m_{W}^{2}\right)\left(m_{j}^{2}-m_{W}^{2}\right)} \\
& -\frac{2\left(m_{i}^{2} m_{j}^{2} m_{W}^{2}+m_{i}^{2} m_{j}^{4}-2 m_{i}^{2} m_{W}^{4}-7 m_{j}^{4} m_{W}^{2}+2 m_{j}^{2} m_{W}^{4}+2 m_{j}^{6}\right) \log \frac{m_{i}^{2}}{m_{j}^{2}}}{\left(m_{i}^{2}-m_{j}^{2}\right)\left(m_{j}^{2}-m_{W}^{2}\right)^{2}} \\
& -\frac{2 m_{W}^{4}\left(17 m_{i}^{2} m_{j}^{2}-10 m_{i}^{2} m_{W}^{2}+5 m_{i}^{4}-7 m_{j}^{2} m_{W}^{2}+2 m_{j}^{4}+2 m_{W}^{4}\right) \log \frac{m_{i}^{2}}{m_{W}^{2}}}{\left(m_{i}^{2}-m_{W}^{2}\right)^{2}\left(m_{j}^{2}-m_{W}^{2}\right)^{2}} \\
& -\frac{2 m_{i}^{2} m_{j}^{2}\left(2 m_{i}^{2} m_{j}^{2}-7 m_{i}^{2} m_{W}^{2}-4 m_{j}^{2} m_{W}^{2}\right) \log \frac{m_{i}^{2}}{m_{W}^{2}}}{\left(m_{i}^{2}-m_{W}^{2}\right)^{2}\left(m_{j}^{2}-m_{W}^{2}\right)^{2}} \\
& F_{2}=-6\left(\frac{1}{\epsilon}+\log \frac{\mu^{2}}{\xi m_{W}^{2}}\right)+2 \frac{m_{j}^{2}}{m_{j}^{2}-\xi m_{W}^{2}} \log \frac{m_{j}^{2}}{\xi m_{W}^{2}} \\
& -\frac{5 m_{i}^{2}\left(m_{j}^{2}-\xi m_{W}^{2}\right)+\xi m_{W}^{2}\left(7 \xi m_{W}^{2}-5 m_{j}^{2}\right)}{\left(m_{i}^{2}-\xi m_{W}^{2}\right)\left(m_{j}^{2}-\xi m_{W}^{2}\right)} \\
& +\frac{2 m_{j}^{2}\left[m_{i}^{2}\left(m_{j}^{2}-\xi m_{W}^{2}\right)-3 \xi m_{j}^{2} m_{W}^{2}+2 m_{j}^{4}\right] \log \frac{m_{i}^{2}}{m_{j}^{2}}}{\left(m_{i}^{2}-m_{j}^{2}\right)\left(m_{j}^{2}-\xi m_{W}^{2}\right)^{2}} \\
& -\frac{2 \xi^{2} m_{j}^{2} m_{W}^{4}\left(2 m_{j}^{2}-3 \xi m_{W}^{2}\right) \log \frac{m_{i}^{2}}{\xi m_{W}^{2}}}{\left(m_{i}^{2}-\xi m_{W}^{2}\right)^{2}\left(m_{j}^{2}-\xi m_{W}^{2}\right)^{2}} \\
& +\frac{2 \xi m_{i}^{2} m_{W}^{2}\left(9 \xi m_{j}^{2} m_{W}^{2}-4 m_{j}^{4}-4 \xi^{2} m_{W}^{4}\right) \log \frac{m_{i}^{2}}{\xi m_{W}^{2}}}{\left(m_{i}^{2}-\xi m_{W}^{2}\right)^{2}\left(m_{j}^{2}-\xi m_{W}^{2}\right)^{2}} \\
& +\frac{2 m_{i}^{4}\left(2 m_{j}^{4}-5 \xi m_{j}^{2} m_{W}^{2}+3 \xi^{2} m_{W}^{4}\right) \log \frac{m_{i}^{2}}{\xi m_{W}^{2}}}{\left(m_{i}^{2}-\xi m_{W}^{2}\right)^{2}\left(m_{j}^{2}-\xi m_{W}^{2}\right)^{2}} .
\end{aligned}
$$


Here we have used dimensional regularization which means the integrals are computed in a $d=4-2 \epsilon$ dimensional spacetime. And the generalization of integration measure $\int \frac{d^{4} k}{(2 \pi)^{4}} \rightarrow \mu^{2 \epsilon} \int \frac{d^{d} k}{(2 \pi)^{d}}$ introduces a dimensional constant $\mu$ which, together with $1 / \epsilon$, should be canceled out in physical results.

We have verified that the above expressions are symmetric under $i \leftrightarrow j$ :

$$
F_{1}=\left.F_{1}\right|_{i \leftrightarrow j}, \quad F_{2}=\left.F_{2}\right|_{i \leftrightarrow j} .
$$

In addition, though $m_{i}^{2}-m_{j}^{2}$ appears in some of the denominators, it does not cause additional divergences when $m_{i} \rightarrow m_{j}$ :

$$
\begin{aligned}
\lim _{m_{j} \rightarrow m_{i}} F_{1}= & 6\left(\frac{1}{\epsilon}+\log \frac{\mu^{2}}{m_{W}^{2}}\right) \\
& +\frac{3 m_{i}^{2} m_{W}^{4}\left[3-8 \log \frac{m_{i}^{2}}{m_{W}^{2}}\right]+\left(3 m_{i}^{4} m_{W}^{2}-m_{i}^{6}\right)\left[1+6 \log \frac{m_{i}^{2}}{m_{W}^{2}}\right]-11 m_{W}^{6}}{\left(m_{i}^{2}-m_{W}^{2}\right)^{3}}, \\
\lim _{m_{j} \rightarrow m_{i}} F_{2}= & -6\left(\frac{1}{\epsilon}+\log \frac{\mu^{2}}{\xi m_{W}^{2}}\right) \\
& +\frac{2 m_{i}^{2}\left(-9 \xi m_{i}^{2} m_{W}^{2}+3 m_{i}^{4}+8 \xi^{2} m_{W}^{4}\right)}{\left(m_{i}^{2}-\xi m_{W}^{2}\right)^{3}} \log \frac{m_{i}^{2}}{\xi m_{W}^{2}} \\
& +\frac{-9 \xi^{2} m_{i}^{2} m_{W}^{4}+\xi m_{i}^{4} m_{W}^{2}+m_{i}^{6}+7 \xi^{3} m_{W}^{6}}{\left(m_{i}^{2}-\xi m_{W}^{2}\right)^{3}} .
\end{aligned}
$$

To obtain the final result of $i \mathcal{M}_{W}$, one needs both eqs. (A.1)-(A.2) and eqs. (A.4)-(A.5) to sum over $i$ and $j$ as it involves cases of $i \neq j$ and $i=j$.

\section{B Some cancellations}

In this appendix, we discuss several noteworthy cancellations in our calculation.

\section{B.1 Cancellation of UV divergences}

As can be seen from eqs. (A.1) and (A.2), both $F_{1}$ and $F_{2}$ contain UV divergences $1 / \epsilon$ in their first terms. When combined together in eq. (3.10), there is obviously a cancellation between the two divergences:

$$
6\left(\frac{1}{\epsilon}+\log \frac{\mu^{2}}{m_{W}^{2}}\right)-6\left(\frac{1}{\epsilon}+\log \frac{\mu^{2}}{\xi m_{W}^{2}}\right)=6 \log \xi .
$$

Further cancellations of $\log \xi$ will be discussed in the next subsection.

Here we would like to address a subtlety concerning UV divergences in the unitarity gauge. If we had naively taken the $\xi \rightarrow \infty$ limit of eq. (3.4) at the beginning of the above calculations, we would get a divergent result because

$$
\lim _{\xi \rightarrow \infty} \Delta_{\mu \nu}^{W}(k)=-i \frac{g_{\mu \nu}-k_{\mu} k_{\nu} / m_{W}^{2}}{k^{2}-m_{W}^{2}},
$$


which corresponds to exactly the $F_{1}$ contribution according to eq. (3.8). And our calculation has shown that the $F_{1}$ contribution itself is UV divergent. We also know that the divergence is actually canceled out by the $F_{2}$ contribution, which however would vanish if $\xi \rightarrow \infty$ had been taken in the naive way. That implies that taking $\xi \rightarrow \infty$ should be after the loop integration. Actually from the second term of (3.8), one can see that when the loop integral contains $\frac{k_{\mu} k_{\nu} / m_{W}^{2}}{k^{2}-\xi m_{W}^{2}}$, the $\xi \rightarrow \infty$ limit does not commute with $k \rightarrow \infty$ in the integral. Taking $\xi \rightarrow \infty$ after the integration can make the large momentum contribution with $k^{2}>\xi m_{W}^{2}$ be included, which is crucial for the UV cancellation.

In other gauges, it is more straightforward to see that $i \mathcal{M}_{W}$ is finite. Taking the Feynman-'t Hooft gauge for example,

$$
\lim _{\xi \rightarrow 1} \Delta_{\mu \nu}^{W}(k)=\frac{-i g_{\mu \nu}}{k^{2}-m_{W}^{2}},
$$

when it is applied to eq. (3.1), using eq. (3.9), the loop integral becomes

$$
\int \frac{d^{4} k}{(2 \pi)^{4}} \gamma_{L}^{\mu} \frac{\not p_{j} \lambda_{j}+\not p_{i} \lambda_{i}}{\left(p_{j}^{2}-m_{j}^{2}\right)\left(p_{i}^{2}-m_{i}^{2}\right)} \gamma_{L}^{\nu} \frac{g_{\mu \nu}}{k^{2}-m_{W}^{2}} \stackrel{\text { large } k}{\longrightarrow}\left(\lambda_{i}+\lambda_{j}\right) \int \frac{d^{4} k}{(2 \pi)^{4}} \frac{\gamma_{L}^{\mu} \not k \gamma_{L \mu}}{k^{6}}
$$

where $\lambda_{j} \equiv m_{i} y_{R}^{j i *}$ and $\lambda_{i} \equiv y_{R}^{j i} m_{j}$. Is is now obvious to see that the loop integral converges because the integrand is proportional to $k^{-5}$ as $k \rightarrow \infty$.

\section{B.2 Cancellation of $\xi$ dependence in $R_{\xi}$ gauges}

The $F_{1}$ contribution is $\xi$ independent. So we are only concerned with $F_{2}$. Let us make a series expansion of $F_{2}$ with respect to $\xi^{-1}$ :

$$
F_{2}=-6\left(\frac{1}{\epsilon}+\log \frac{\mu^{2}}{m_{W}^{2}}\right)-7+6 \log \xi+\mathcal{O}\left(\xi^{-1}\right) .
$$

Usually in $R_{\xi}$ gauges, it is expected that the $\xi$ dependence of a $W^{ \pm}$diagram is canceled by a similar diagram with $W^{ \pm}$replaced by its Goldstone boson $H^{ \pm}$. In our case, it would be the diagram in figure 5 . However, a straightforward calculation shows that the amplitude of this diagram is

$$
i \mathcal{M}_{H^{ \pm}} \propto m_{\ell} \frac{m_{j} y_{R}^{i j}+m_{i} y_{R}^{i j *}}{m_{W}^{2} \xi}+\mathcal{O}\left(\xi^{-2}\right),
$$

which is impossible to cancel the $\log \xi$ term in eq. (B.5) when $\xi$ increases to sufficiently large values.

This problem is essentially related to the completeness of the model. For an arbitrary matrix of $y_{R}^{i j}$, indeed the result would be gauge dependent and the $\log \xi$ term remains for each case of $(i, j)=(1,1),(1,4),(4,1)$, and $(4,4)$. However, in section 2 we have shown that the elements in $y_{R}^{i j}$ are correlated by active-sterile neutrino mixing — see eq. (2.19). Besides, $g_{W}^{i}$ also depends on the mixing - see eq. (2.20). As a consequence, when summing up the contributions of both light and heavy neutrinos in eq. (3.10), the $\log \xi$ term cancels out because

$$
\sum_{i, j} G^{i j} 6 \log \xi=0
$$




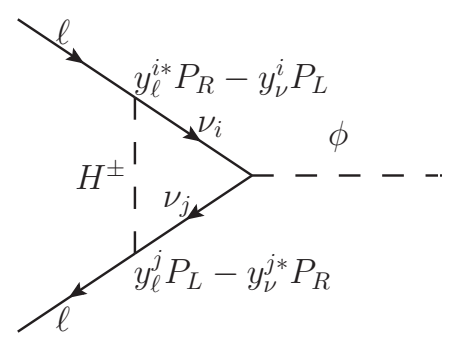

Figure 5. The Goldstone boson diagram that complements the $W^{ \pm}$diagram to cancel the $\xi$ dependence in $R_{\xi}$ gauges.

Therefore, the $\log \xi$ term can be safely ignored when computing the full amplitude. Actually if we inspect $G^{i j}$ in the chiral basis, the cancellation is more manifest. From eq. (3.11), we can express $\sum_{i, j} G^{i j}$ in the matrix form:

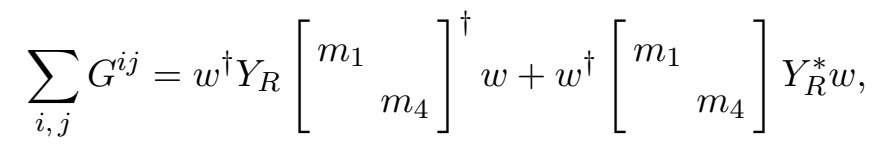

where $w$ and $Y_{R}$ are the vector and matrix of $g_{W}^{i}$ and $y_{R}^{i j}$ in eqs. (2.20) and (2.19) respectively. They are transformed from the chiral basis by:

$$
w \equiv \frac{g}{\sqrt{2}} U^{T}\left[\begin{array}{l}
1 \\
0
\end{array}\right], \quad Y_{R} \equiv y_{R} U^{T}\left[\begin{array}{ll}
0 & \\
& 1
\end{array}\right] U .
$$

Therefore, in the chiral basis, we have

$$
\begin{aligned}
\sum_{i, j} G^{i j} & \propto(1,0) U^{*}\left\{U^{T}\left[\begin{array}{l}
0 \\
1
\end{array}\right] U U^{\dagger} M_{2 \nu}^{*} U^{*}+U^{T} M_{\nu} U U^{\dagger}\left[\begin{array}{ll}
0 & \\
& 1
\end{array}\right] U^{*}\right\} U^{T}\left[\begin{array}{l}
1 \\
0
\end{array}\right] \\
& =(1,0)\left\{\left[\begin{array}{ll}
0 \\
1
\end{array}\right] M_{2 \nu}^{*}+M_{2 \nu}\left[\begin{array}{l}
0 \\
1
\end{array}\right]\right\}\left[\begin{array}{l}
1 \\
0
\end{array}\right] \\
& =0 .
\end{aligned}
$$

Here $M_{2 \nu}$ is the neutrino mass matrix in eq. (2.10). It shows that the vanishing product of $w$ and $Y_{R}$ (more specifically, $w^{\dagger} Y_{R}=0$ and $Y_{R}^{\dagger} w=0$ ), which is due to the absence of $W^{ \pm}-\nu_{R}$ and $\phi-\nu_{L}$ couplings, leads to $\sum_{i, j} G^{i j}=0$.

\section{B.3 GIM-like cancellation}

If all the neutrino masses (including heavy ones) are much smaller than $m_{W}$, when summing over $i$ and $j$ in eq. (3.10), the leading-order contribution vanishes in a way similar to the Glashow-Iliopoulos-Maiani (GIM) mechanism [82]. At the next-to-leading order (NLO), a nonzero result can be obtained. But in case of zero mass splitting of neutrinos, the NLO contribution would vanish again. This is also similar to the GIM cancellation, where if $u$ and $c$ quarks were of equal mass, the $K^{0} \rightarrow \overline{K^{0}}$ amplitude would be zero. 
Let us compute $i \mathcal{M}_{W}$ in the unitarity gauge. The preceding discussion in section B.2 concludes that the $\log \xi$ term can be safely ignored in this complete model. Hence we define

$$
F_{12} \equiv \lim _{\xi \rightarrow \infty}\left(F_{1}+F_{2}-6 \log \xi\right),
$$

which is finite. Then $i \mathcal{M}_{W}$ in the unitarity gauge can be computed by:

$$
i \mathcal{M}_{W}=i \overline{u\left(p_{2}\right)} u\left(p_{1}\right) \sum_{i, j} \frac{m_{\ell} G^{i j} F_{12}\left(m_{i}, m_{j}\right)}{256 \pi^{2} m_{W}^{2}} .
$$

Now if we assume $m_{W} \gg m_{i}$ and $m_{j}, F_{12}$ can be expanded as follows:

$$
F_{12}=4+\frac{6 m_{i}^{4}\left(1+4 \log \frac{m_{i}}{m_{W}}\right)-6 m_{j}^{4}\left(1+4 \log \frac{m_{j}}{m_{W}}\right)}{m_{W}^{2}\left(m_{i}^{2}-m_{j}^{2}\right)}+\mathcal{O}\left(\frac{m_{i, j}^{4}}{m_{W}^{4}}\right) .
$$

The constant term in $F_{12}$ does not contribute to eq. (B.12) due to $\sum_{i, j} G^{i j}=0$. So the leading-order contribution to $i \mathcal{M}_{W}$ vanishes. Only the second or higher-order terms in eq. (B.13) further suppressed by $m_{i, j}^{2} / m_{W}^{2}$ contribute to nonzero $i \mathcal{M}_{W}$.

Plugging eq. (B.13) into eq. (B.12) and using the explicit form of $G^{i j}$ in eq. (3.11), we obtain

$$
\frac{i \mathcal{M}_{W}}{i \overline{u\left(p_{2}\right)} u\left(p_{1}\right)} \approx \frac{3 g^{2} m_{\ell} s_{\theta}^{2} c_{\theta}^{2}\left(y_{R}+y_{R}^{*}\right)\left[4\left(2 m_{1}+m_{4}\right) m_{4}^{3} \log \left(\frac{m_{4}}{m_{W}}\right)+3 m_{4}^{4}+4 m_{1} m_{4}^{3}-(1 \leftrightarrow 4)\right]}{256 \pi^{2} m_{W}^{4}\left(m_{1}+m_{4}\right)} .
$$

Note that the expression in the square bracket is antisymmetric under the interchange of $m_{1}$ and $m_{4}$. Therefore if the mass splitting $m_{1}-m_{4}$ is zero, the NLO contribution vanishes as well, similar to the GIM cancellation.

In Type I seesaw, the scale of heavy neutrino masses is often assumed to be much higher than the electroweak scale. Hence a more likely scenario is $m_{4} \gg m_{W} \gg m_{1}$. For such a hierarchy, there is no GIM-like cancellation, as we shall show below.

First, we need to expand $F_{12}$ in other regimes. If the diagram contains heavy neutrinos running in the loop, we expand it with respect to $m_{W}$ :

$$
F_{12} \approx \frac{2 m_{j}^{2}\left[1-6 \log \left(\frac{m_{W}}{m_{j}}\right)\right]-2 m_{i}^{2}\left[1-6 \log \left(\frac{m_{W}}{m_{i}}\right)\right]}{m_{i}^{2}-m_{j}^{2}}, \text { for } m_{W} \ll m_{i}, m_{j} .
$$

If the diagram contains one light and one heavy neutrinos, we have

$$
F_{12} \approx-2+\left[12 \log \left(\frac{m_{W}}{m_{j}}\right)-\frac{6 m_{W}^{2}}{m_{j}^{2}}\right]\left(1+\frac{m_{i}^{2}}{m_{j}^{2}}\right)-\frac{6 m_{i}^{2}}{m_{j}^{2}}, \text { for } m_{i} \ll m_{W} \ll m_{j} .
$$

For $m_{j} \ll m_{W} \ll m_{i}$, the result can be obtained by an interchange of $i$ and $j$ in eq. (B.16).

Combining the results in eqs. (B.13), (B.15), and (B.16), we sum over $i$ and $j$ in eq. (B.12), which gives:

$$
\frac{i \mathcal{M}_{W}}{i \overline{u\left(p_{2}\right)} u\left(p_{1}\right)}=-3 g^{2} m_{\ell} m^{3} s_{\theta}^{2} \operatorname{Re}\left(y_{R}\right) \frac{m^{4}-4 m^{2} m_{W}^{2}+m_{W}^{4}\left(3+2 \log \frac{m^{2}}{m_{W}^{2}}\right)}{128 \pi^{2} m_{W}^{2}\left(m^{2}-m_{W}^{2}\right)^{3}}+\mathcal{O}\left(s_{\theta}^{4}\right),
$$


where $m \equiv \sqrt{m_{1}^{2}+m_{4}^{2}}$. Now taking $G_{F}=\sqrt{2} g^{2} /\left(8 m_{W}^{2}\right), m s_{\theta}^{2}=m_{1}$, and $m \gg m_{W}$, we obtain the result in eqs. (3.12) and (3.13).

\section{Pseudo-scalar couplings}

As mentioned in section 3, the $W$ diagram in figure 1 leads to loop-induced couplings of both scalar $(\bar{\psi} \phi \psi)$ and pseudo-scalar $\left(\bar{\psi} i \gamma^{5} \phi \psi\right)$ forms. The $Z$ diagram leads to only pseudo-scalar couplings. Although the pseudo-scalar couplings are not relevant to the phenomenology considered in this work, we would like to present our calculation of the pseudo-scalar couplings in this appendix.

Let us first compute the $Z$ diagram. Starting from eq. (3.2), we have:

$$
\operatorname{tr}\left[-g_{Z}^{i j} \gamma_{L}^{\nu} \Delta_{j}\left(p_{j}\right) \frac{y_{R}^{j i} P_{L}+y_{R}^{j i *} P_{R}}{2} \Delta_{i}\left(p_{i}\right)\right]=g_{Z}^{i j} \frac{m_{j} y_{R}^{j i} p_{i}^{\nu}+m_{i} y_{R}^{j i *} p_{j}^{\nu}}{\left(p_{j}^{2}-m_{j}^{2}\right)\left(p_{i}^{2}-m_{i}^{2}\right)} .
$$

So eq. (3.2) can be written as

$$
i \mathcal{M}_{Z}=(i)^{3} \int \frac{d^{4} p_{i}}{(2 \pi)^{4}} \overline{u\left(p_{2}\right)} g_{Z}^{(\ell)} \gamma_{L}^{\mu} u\left(p_{1}\right) g_{Z}^{i j} \frac{m_{j} y_{R}^{j i} p_{i}^{\nu}+m_{i} y_{R}^{j i *} p_{j}^{\nu}}{\left(p_{j}^{2}-m_{j}^{2}\right)\left(p_{i}^{2}-m_{i}^{2}\right)} \Delta_{\mu \nu}^{Z}(q) .
$$

In the limit of $q \rightarrow 0$,

$$
\Delta_{\mu \nu}^{Z}(q) \rightarrow \frac{i g_{\mu \nu}}{m_{Z}^{2}}
$$

we replace $p_{j}$ with $p_{i}+q$ and extract the $p_{i}$-independent part out of the loop integral:

$$
i \mathcal{M}_{Z}=\frac{i}{16 \pi^{2}} \overline{u\left(p_{2}\right)} g_{Z}^{(\ell)} \gamma_{L \mu} u\left(p_{1}\right) \frac{g_{Z}^{i j}}{m_{Z}^{2}} I_{i j}^{\mu}(q),
$$

where the loop integral $I^{\mu}(q)$ reads:

$$
I_{i j}^{\mu}(q) \equiv\left[\frac{i}{16 \pi^{2}}\right]^{-1} \int \frac{d^{4} p_{i}}{(2 \pi)^{4}} \frac{m_{j} y_{R}^{j i} p_{i}^{\mu}+m_{i} y_{R}^{j i *}\left(p_{i}^{\mu}+q^{\mu}\right)}{\left[\left(p_{i}^{\mu}+q^{\mu}\right)^{2}-m_{j}^{2}\right]\left(p_{i}^{2}-m_{i}^{2}\right)} .
$$

Performing the loop integration, we get

$$
\begin{aligned}
I_{i j}^{\mu}(q)= & q^{\mu} \frac{-m_{j} y_{R}^{j i}+m_{i} y_{R}^{j i *}}{2}\left(\frac{1}{\epsilon}+\log \mu^{2}-\log m_{j}^{2}\right) \\
& +q^{\mu} \frac{y_{R}^{j i} m_{j}\left(m_{j}^{2}-3 m_{i}^{2}\right)+y_{R}^{j i *} m_{i}\left(m_{i}^{2}-3 m_{j}^{2}\right)}{4\left(m_{i}^{2}-m_{j}^{2}\right)} \\
& +q^{\mu} \frac{-y_{R}^{j i} m_{j} m_{i}+y_{R}^{j i *}\left(m_{i}^{2}-2 m_{j}^{2}\right)}{2\left(m_{i}^{2}-m_{j}^{2}\right)^{2}} m_{i}^{3} \log \frac{m_{i}^{2}}{m_{j}^{2}} \\
& +q^{\mu} \mathcal{O}\left(q^{2}\right) .
\end{aligned}
$$

Next, we sum over $i$ and $j$ and expand the result in $s_{\theta}$ :

$$
\sum_{i, j} g_{Z}^{i j} I_{i j}^{\mu}(q) \approx q^{\mu} \frac{y_{R}-y_{R}^{*}}{4} m s_{\theta}^{2}+\mathcal{O}\left(s_{\theta}^{3}\right)
$$


The UV divergence cancels out in the summation because

$$
\sum_{i, j} g_{Z}^{i j} m_{j} y_{R}^{j i}=\sum_{i, j} g_{Z}^{i j} m_{i} y_{R}^{j i *}=0
$$

which can be proven straightforwardly from eqs. (2.19).

Plugging eq. (C.6) into eq. (C.3), we obtain

$$
\begin{aligned}
i \mathcal{M}_{Z} & =i \frac{g_{Z}^{(\ell)} m_{1}\left(y_{R}-y_{R}^{*}\right)}{64 \pi^{2} m_{Z}^{2}} \overline{u\left(p_{2}\right)} \phi P_{L} u\left(p_{1}\right) \\
& =-i \frac{g_{Z}^{(\ell)} m_{\ell} m_{1}\left(y_{R}-y_{R}^{*}\right)}{64 \pi^{2} m_{Z}^{2}} \overline{u\left(p_{2}\right)} \gamma^{5} u\left(p_{1}\right),
\end{aligned}
$$

where in the second step we have used eq. (3.7).

Computing the pseudo-scalar coupling from the $W$ diagram is similar, except that the bilinear $\overline{u\left(p_{2}\right)}\left(C_{1} \not p_{1}+C_{2} \not p_{2}\right) P_{L} u\left(p_{1}\right)$ where $C_{1}$ and $C_{2}$ are different scalar quantities cannot be converted to $\overline{u\left(p_{2}\right)} \phi P_{L} u\left(p_{1}\right)$. It actually contributes to both scalar and pseudo-scalar couplings because

$$
\begin{aligned}
\overline{u\left(p_{2}\right)}\left(C_{1} \not p_{1}+C_{2} \not p_{2}\right) P_{L} u\left(p_{1}\right) & =m_{\ell} \overline{u\left(p_{2}\right)}\left(C_{1} P_{R}+C_{2} P_{L}\right) u\left(p_{1}\right) \\
& =m_{\ell} \frac{C_{1}+C_{2}}{2} \overline{u\left(p_{2}\right)} u\left(p_{1}\right)+m_{\ell} \frac{C_{1}-C_{2}}{2} \overline{u\left(p_{2}\right)} \gamma^{5} u\left(p_{1}\right) .
\end{aligned}
$$

With this detail being noted, the calculation is straightforward and gives:

$$
i \mathcal{M}_{W} \approx-i \frac{G_{F} m_{1} m_{\ell}}{8 \sqrt{2} \pi^{2}} \overline{u\left(p_{2}\right)}\left[\frac{3}{4}\left(y_{R}+y_{R}^{*}\right)+\frac{1}{2}\left(y_{R}-y_{R}^{*}\right) \gamma^{5}\right] u\left(p_{1}\right) .
$$

The first and second terms in the square bracket give rise to the loop-induced scalar and pseudo-scalar couplings respectively. The former has been considered in section 3 . The latter and the $i \mathcal{M}_{Z}$ amplitude in eq. (C.9) lead to the following pseudo-scalar interaction:

$$
\mathcal{L} \supset \lambda_{\phi \ell \ell}^{(W)} \phi \overline{\psi_{\ell}} i \gamma^{5} \psi_{\ell}+\lambda_{\phi \ell \ell}^{(Z)} \phi \overline{\psi_{\ell}} i \gamma^{5} \psi_{\ell}
$$

where

$$
\lambda_{\phi \ell \ell}^{(W)}=-\frac{G_{F} m_{1} m_{\ell} \operatorname{Im}\left(y_{R}\right)}{8 \sqrt{2} \pi^{2}}, \lambda_{\phi \ell \ell}^{(Z)}=-\frac{g_{Z}^{(\ell)} m_{1} m_{\ell} \operatorname{Im}\left(y_{R}\right)}{32 \pi^{2} m_{Z}^{2}} .
$$

\section{Generalization to three flavors}

In this appendix, we present the detailed three-flavor analysis.

The $6 \times 6$ symmetric mass matrix can be diagonalized by a $6 \times 6$ unitary matrix $U$ :

$$
U^{T} M_{6 \nu} U=\operatorname{diag}\left(m_{1}, m_{2}, m_{3}, \cdots, m_{6}\right) \equiv M_{d} .
$$

The neutrino flavor basis and the mass basis are connected by

$$
\left(\begin{array}{c}
\nu_{L} \\
\nu_{R}
\end{array}\right)=U\left(\begin{array}{c}
\nu_{1} \\
\vdots \\
\nu_{6}
\end{array}\right)
$$


where both $\nu_{L}$ and $\nu_{R}$ are $3 \times 1$ vectors. First, we convert the gauge interaction $\frac{g}{\sqrt{2}} W_{\mu}^{-} \ell_{L}^{\dagger} \bar{\sigma}^{\mu} \nu_{L}$ from the flavor basis to the mass basis:

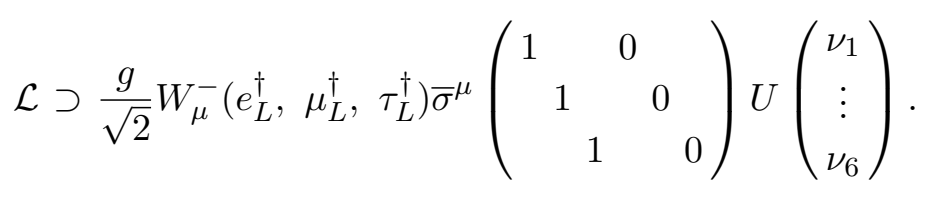

This generalizes $g_{W}^{i}$ in eq. (2.20) from a $1 \times 2$ vector to a $3 \times 6$ matrix:

$$
g_{W}^{\ell i}=\frac{g}{\sqrt{2}} U_{\ell i}, \quad(\ell=e, \mu, \tau, \text { and } i=1 \cdots 6) .
$$

Next, we perform a similar transformation for the Yukawa interactions of $\nu_{R}$ :

$$
\frac{1}{2} \phi \nu_{R}^{T} Y_{R}^{0} \nu_{R}=\frac{\phi}{2}\left(\nu_{1}, \cdots, \nu_{6}\right) U^{T}\left(\begin{array}{ccc}
0 & & \\
& 0 & \\
& 0 & \\
& & Y_{R}^{0}
\end{array}\right) U\left(\begin{array}{c}
\nu_{1} \\
\vdots \\
\nu_{6}
\end{array}\right) \equiv \frac{\phi}{2}\left(\nu_{1} \cdots \nu_{6}\right) Y_{R}\left(\begin{array}{c}
\nu_{1} \\
\vdots \\
\nu_{6}
\end{array}\right),
$$

where the $6 \times 6$ matrix $Y_{R}$ is a generalization of the $2 \times 2 y_{R}^{i j}$ matrix in eq. (2.19).

The generalization of $G^{i j}$ is quite straightforward. By replacing $g_{W}^{i}$ and $y_{R}^{i j}$ in eq. (3.11) with $g_{W}^{\ell i}$ and $Y_{R}^{i j}$, we get:

$$
G^{i j}=g_{W}^{\ell *} g_{W}^{\ell j}\left(m_{j} Y_{R}^{i j}+m_{i} Y_{R}^{i j *}\right)
$$

As for $F_{1}$ and $F_{2}$, the expressions in eqs. (A.1) and (A.2) can be used directly except that now $i$ and $j$ run from 1 to 6 instead of 1 and 4 .

With the generalized $G^{i j}, F_{1}$ and $F_{2}$, it is straightforward to get the loop-induced effective Yukawa coupling in eq. (4.3).

Note that any constant terms in $F_{12}$ can be ignored because

$$
\begin{aligned}
& \sum_{i, j} U_{\ell i}^{*} U_{\ell j}\left(Y_{R} M_{d}+M_{d} Y_{R}^{\dagger}\right)_{i j} \\
& =\left[\left(\begin{array}{cc}
1_{3 \times 3} & \\
& 0_{3 \times 3}
\end{array}\right) U^{*} U^{T}\left(\begin{array}{ll}
0_{3 \times 3} & \\
& Y_{R}^{0}
\end{array}\right) U M_{d} U^{T}\left(\begin{array}{cc}
1_{3 \times 3} & \\
& 0_{3 \times 3}
\end{array}\right)+\text { h.c. }\right]_{\ell \ell} \\
& =\left[\left(\begin{array}{cc}
1_{3 \times 3} & \\
& 0_{3 \times 3}
\end{array}\right)\left(\begin{array}{cc}
0_{3 \times 3} & \\
& Y_{R}^{0}
\end{array}\right) M_{6 \nu}^{*}\left(\begin{array}{cc}
1_{3 \times 3} & \\
& 0_{3 \times 3}
\end{array}\right)+\text { h.c. }\right]_{\ell \ell} \\
& =0
\end{aligned}
$$

which is similar to eq. (B.10).

Eq. (4.3) applies for the most general $3 \nu_{L}+3 \nu_{R}$ scenario. Although its dependence on the PMNS matrix and light neutrino masses is not manifest, each quantity in eq. (4.3) can be readily evaluated using numerical methods.

Below we would like to discuss a special case in which eq. (4.3) can be further simplified and expressed in the Casas-Ibarra parametrization [54]. 
If $M_{R}$ and $Y_{R}^{0}$ can be simultaneously diagonalized and $m_{1,2,3} \ll m_{4,5,6}$, without loss of generality we can assume $M_{R}$ and $Y_{R}^{0}$ are diagonal and $m_{D}$ can be expressed, according to the Casas-Ibarra parametrization, as

$$
m_{D}=i U_{L}^{*} \sqrt{m_{\nu}^{d}} R^{T} \sqrt{M_{R}}
$$

where $U_{L}, m_{\nu}^{d}$ and $R$ have been defined in section 4 .

Then the full $6 \times 6$ mixing matrix $U$ can be approximately decomposed as

$$
U \approx\left(\begin{array}{cc}
U_{L} & \\
& 1_{3 \times 3}
\end{array}\right)\left(\begin{array}{cc}
1_{3 \times 3} & -i T \\
-i T^{\dagger} & 1_{3 \times 3}
\end{array}\right)
$$

where

$$
T \equiv \sqrt{m_{\nu}^{d}} R^{\dagger} \sqrt{M_{R}^{-1}}
$$

From eqs. (D.8) and (D.10), we have

$$
m_{D} M_{R}^{-1}=i U_{L}^{*} T^{*}
$$

In the mass basis, the Yukawa coupling matrix $Y_{R}$, defined in eq. (D.5), now reads

$$
Y_{R} \approx\left(\begin{array}{cc}
\mathcal{O}\left(T^{2}\right) & -i T^{*} Y_{R}^{0} \\
-i Y_{R}^{0} T^{\dagger} & Y_{R}^{0}
\end{array}\right)
$$

Next, we need to compute $F_{12}\left(m_{i}, m_{j}\right)$ in eq. (4.3). Assuming $m_{4,5,6} \gg m_{W} \gg m_{1,2,3}$, the result is

$$
F_{12} \approx\left(\begin{array}{cccccc}
4 & 4 & 4 & f_{4}-2 & f_{5}-2 & f_{6}-2 \\
4 & 4 & 4 & f_{4}-2 & f_{5}-2 & f_{6}-2 \\
4 & 4 & 4 & f_{4}-2 & f_{5}-2 & f_{6}-2 \\
f_{4}-2 & f_{4}-2 & f_{4}-2 & f_{4}-8 & . & . \\
f_{5}-2 & f_{5}-2 & f_{5}-2 & . & f_{5}-8 & . \\
f_{6}-2 & f_{6}-2 & f_{6}-2 & . & . & f_{6}-8
\end{array}\right),
$$

where $f_{i} \equiv 12 \log \frac{m_{i}}{m_{W}}$ and "." denotes more complicated expressions which are irrelevant to our calculation.

By introducing the following matrix:

$$
H_{i j} \equiv\left(Y_{R} M_{d}+M_{d} Y_{R}^{\dagger}\right)_{i j} F_{12}\left(m_{i}, m_{j}\right)
$$

we can reformulate eq. (4.3) as

$$
y_{\phi \ell \ell}=\frac{G_{F} m_{\ell}}{64 \sqrt{2} \pi^{2}}\left[U^{*} H U^{T}\right]_{\ell \ell} .
$$

Combining eqs. (D.11) and (D.12), we obtain the $H$ matrix:

$$
H=\left(\begin{array}{cc}
\mathcal{O}\left(T^{4}\right) & -i T^{*} Y_{R}^{0} M_{R} D_{f}+\mathcal{O}\left(T^{3}\right) \\
\left(-i T^{*} Y_{R}^{0} M_{R} D_{f}\right)^{\dagger}+\mathcal{O}\left(T^{3}\right) & Y_{R}^{0} M_{R}\left(D_{f}-6 I_{3}\right)+\text { h.c. }
\end{array}\right),
$$

where $D_{f} \equiv \operatorname{diag}\left(f_{4}-2, f_{5}-2, f_{6}-2\right)$ and $I_{3}$ is a $3 \times 3$ identity matrix. Now supplying all the matrices required by eq. (D.14), we obtain the results in eqs. (4.5) and (4.6). 
Open Access. This article is distributed under the terms of the Creative Commons Attribution License (CC-BY 4.0), which permits any use, distribution and reproduction in any medium, provided the original author(s) and source are credited.

\section{References}

[1] T. Asaka and M. Shaposhnikov, The $\nu M S M$, dark matter and baryon asymmetry of the universe, Phys. Lett. B 620 (2005) 17 [hep-ph/0505013] [INSPIRE].

[2] T. Asaka, S. Blanchet and M. Shaposhnikov, The nuMSM, dark matter and neutrino masses, Phys. Lett. B 631 (2005) 151 [hep-ph/0503065] [INSPIRE].

[3] T. Alanne, T. Hugle, M. Platscher and K. Schmitz, Low-scale leptogenesis assisted by a real scalar singlet, JCAP 03 (2019) 037 [arXiv: 1812.04421] [INSPIRE].

[4] P. Minkowski, $\mu \rightarrow e \gamma$ at a Rate of One Out of $10^{9}$ Muon Decays?, Phys. Lett. B 67 (1977) 421 [INSPIRE].

[5] T. Yanagida, Horizontal gauge symmetry and masses of neutrinos, in Proceedings of the Workshop on the Unified Theory and the Baryon Number in the Universe, Tsukuba Japan (1979), KEK Report 79-18-95 (1979).

[6] M. Gell-Mann, P. Ramond and R. Slansky, Complex Spinors and Unified Theories, Conf. Proc. C $\mathbf{7 9 0 9 2 7}$ (1979) 315 [arXiv: 1306.4669] [INSPIRE].

[7] S. Glashow, The future of elementary particle physics, NATO Sci. Ser. B 61 (1979) 687.

[8] R. Mohapatra and G. Senjanovic, Neutrino mass and spontaneous parity nonconservation, Phys. Rev. Lett. 44 (1980) 912 [INSPIRE].

[9] S.G. Turyshev and J.G. Williams, Space-based tests of gravity with laser ranging, Int. J. Mod. Phys. D 16 (2007) 2165 [gr-qc/0611095] [InSPIRE].

[10] S. Schlamminger, K.-Y. Choi, T.A. Wagner, J.H. Gundlach and E.G. Adelberger, Test of the equivalence principle using a rotating torsion balance, Phys. Rev. Lett. 100 (2008) 041101 [arXiv: 0712.0607] [INSPIRE].

[11] B.R. Heckel, E.G. Adelberger, C.E. Cramer, T.S. Cook, S. Schlamminger and U. Schmidt, Preferred-Frame and CP-Violation Tests with Polarized Electrons, Phys. Rev. D 78 (2008) 092006 [arXiv: 0808.2673] [INSPIRE].

[12] LIGO Scientific and VIRGo collaborations, GW170817: Observation of Gravitational Waves from a Binary Neutron Star Inspiral, Phys. Rev. Lett. 119 (2017) 161101 [arXiv: 1710.05832] [INSPIRE].

[13] LiGO Scientific and Virgo collaborations, GW190814: Gravitational Waves from the Coalescence of a 23 Solar Mass Black Hole with a 2.6 Solar Mass Compact Object, Astrophys. J. Lett. 896 (2020) L44 [arXiv:2006.12611] [INSPIRE].

[14] D. Croon, A.E. Nelson, C. Sun, D.G.E. Walker and Z.-Z. Xianyu, Hidden-Sector Spectroscopy with Gravitational Waves from Binary Neutron Stars, Astrophys. J. Lett. 858 (2018) L2 [arXiv:1711.02096] [INSPIRE].

[15] M. Baryakhtar, R. Lasenby and M. Teo, Black Hole Superradiance Signatures of Ultralight Vectors, Phys. Rev. D 96 (2017) 035019 [arXiv:1704.05081] [INSPIRE].

[16] L. Sagunski et al., Neutron star mergers as a probe of modifications of general relativity with finite-range scalar forces, Phys. Rev. D 97 (2018) 064016 [arXiv:1709.06634] [INSPIRE]. 
[17] A. Hook and J. Huang, Probing axions with neutron star inspirals and other stellar processes, JHEP 06 (2018) 036 [arXiv:1708.08464] [INSPIRE].

[18] J. Huang, M.C. Johnson, L. Sagunski, M. Sakellariadou and J. Zhang, Prospects for axion searches with Advanced LIGO through binary mergers, Phys. Rev. D 99 (2019) 063013 [arXiv: 1807.02133] [INSPIRE].

[19] J. Kopp, R. Laha, T. Opferkuch and W. Shepherd, Cuckoo's eggs in neutron stars: can LIGO hear chirps from the dark sector?, JHEP 11 (2018) 096 [arXiv: 1807.02527] [INSPIRE].

[20] S. Alexander, E. McDonough, R. Sims and N. Yunes, Hidden-Sector Modifications to Gravitational Waves From Binary Inspirals, Class. Quant. Grav. 35 (2018) 235012 [arXiv: 1808.05286] [INSPIRE].

[21] H.G. Choi and S. Jung, New probe of dark matter-induced fifth force with neutron star inspirals, Phys. Rev. D 99 (2019) 015013 [arXiv:1810.01421] [InSPIRE].

[22] M. Fabbrichesi and A. Urbano, Charged neutron stars and observational tests of a dark force weaker than gravity, JCAP 06 (2020) 007 [arXiv: 1902.07914] [INSPIRE].

[23] B.C. Seymour and K. Yagi, Probing Massive Scalar Fields from a Pulsar in a Stellar Triple System, Class. Quant. Grav. 37 (2020) 145008 [arXiv: 1908. 03353] [INSPIRE].

[24] J.A. Dror, R. Laha and T. Opferkuch, Probing muonic forces with neutron star binaries, Phys. Rev. D 102 (2020) 023005 [arXiv:1909.12845] [INSPIRE].

[25] Y. Chikashige, R.N. Mohapatra and R.D. Peccei, Are There Real Goldstone Bosons Associated with Broken Lepton Number?, Phys. Lett. B 98 (1981) 265 [INSPIRE].

[26] C. Garcia-Cely and J. Heeck, Neutrino Lines from Majoron Dark Matter, JHEP 05 (2017) 102 [arXiv: 1701.07209] [INSPIRE].

[27] J. Heeck and H.H. Patel, Majoron at two loops, Phys. Rev. D 100 (2019) 095015 [arXiv: 1909.02029] [INSPIRE].

[28] A.S. Joshipura and S. Mohanty, Constraints on flavor dependent long range forces from atmospheric neutrino observations at Super-Kamiokande, Phys. Lett. B 584 (2004) 103 [hep-ph/0310210] [INSPIRE].

[29] J.A. Grifols and E. Masso, Neutrino oscillations in the sun probe long range leptonic forces, Phys. Lett. B 579 (2004) 123 [hep-ph/0311141] [INSPIRE].

[30] A. Bandyopadhyay, A. Dighe and A.S. Joshipura, Constraints on flavor-dependent long range forces from solar neutrinos and KamLAND, Phys. Rev. D 75 (2007) 093005 [hep-ph/0610263] [INSPIRE].

[31] M.C. Gonzalez-Garcia, P.C. de Holanda, E. Masso and R. Zukanovich Funchal, Probing long-range leptonic forces with solar and reactor neutrinos, JCAP 01 (2007) 005 [hep-ph/0609094] [INSPIRE].

[32] A.E. Nelson and J. Walsh, Short Baseline Neutrino Oscillations and a New Light Gauge Boson, Phys. Rev. D 77 (2008) 033001 [arXiv:0711.1363] [INSPIRE].

[33] M.C. Gonzalez-Garcia, P.C. de Holanda and R. Zukanovich Funchal, Constraints from Solar and Reactor Neutrinos on Unparticle Long-Range Forces, JCAP 06 (2008) 019 [arXiv: 0803.1180] [INSPIRE].

[34] A. Samanta, Long-range Forces: Atmospheric Neutrino Oscillation at a magnetized Detector, JCAP 09 (2011) 010 [arXiv: 1001.5344] [InSPIRE]. 
[35] J. Heeck and W. Rodejohann, Gauged $L_{\mu}-L_{\tau}$ and different Muon Neutrino and Anti-Neutrino Oscillations: MINOS and beyond, J. Phys. G 38 (2011) 085005 [arXiv: 1007.2655] [INSPIRE].

[36] H. Davoudiasl, H.-S. Lee and W.J. Marciano, Long-Range Lepton Flavor Interactions and Neutrino Oscillations, Phys. Rev. D 84 (2011) 013009 [arXiv:1102.5352] [INSPIRE].

[37] J. Heeck, Unbroken B - L symmetry, Phys. Lett. B 739 (2014) 256 [arXiv:1408.6845] [INSPIRE].

[38] S.S. Chatterjee, A. Dasgupta and S.K. Agarwalla, Exploring Flavor-Dependent Long-Range Forces in Long-Baseline Neutrino Oscillation Experiments, JHEP 12 (2015) 167 [arXiv: 1509.03517] [INSPIRE].

[39] M. Bustamante and S.K. Agarwalla, Universe's Worth of Electrons to Probe Long-Range Interactions of High-Energy Astrophysical Neutrinos, Phys. Rev. Lett. 122 (2019) 061103 [arXiv: 1808.02042] [INSPIRE].

[40] A. Khatun, T. Thakore and S. Kumar Agarwalla, Can INO be Sensitive to Flavor-Dependent Long-Range Forces?, JHEP 04 (2018) 023 [arXiv:1801.00949] [INSPIRE].

[41] M.B. Wise and Y. Zhang, Lepton Flavorful Fifth Force and Depth-dependent Neutrino Matter Interactions, JHEP 06 (2018) 053 [arXiv: 1803.00591] [INSPIRE].

[42] G. Krnjaic, P.A.N. Machado and L. Necib, Distorted neutrino oscillations from time varying cosmic fields, Phys. Rev. D 97 (2018) 075017 [arXiv:1705.06740] [INSPIRE].

[43] A. Berlin, Neutrino Oscillations as a Probe of Light Scalar Dark Matter, Phys. Rev. Lett. 117 (2016) 231801 [arXiv:1608.01307] [INSPIRE].

[44] V. Brdar, J. Kopp, J. Liu, P. Prass and X.-P. Wang, Fuzzy dark matter and nonstandard neutrino interactions, Phys. Rev. D 97 (2018) 043001 [arXiv:1705.09455] [INSPIRE].

[45] A.Y. Smirnov and X.-J. Xu, Wolfenstein potentials for neutrinos induced by ultra-light mediators, JHEP 12 (2019) 046 [arXiv:1909.07505] [INSPIRE].

[46] K.S. Babu, G. Chauhan and P.S. Bhupal Dev, Neutrino nonstandard interactions via light scalars in the Earth, Sun, supernovae, and the early Universe, Phys. Rev. D 101 (2020) 095029 [arXiv: 1912.13488] [INSPIRE].

[47] H.K. Dreiner, H.E. Haber and S.P. Martin, Two-component spinor techniques and Feynman rules for quantum field theory and supersymmetry, Phys. Rept. 494 (2010) 1 [arXiv: 0812.1594] [INSPIRE].

[48] F. Wilczek, Axions and Family Symmetry Breaking, Phys. Rev. Lett. 49 (1982) 1549 [INSPIRE].

[49] J.E. Moody and F. Wilczek, New macroscopic forces?, Phys. Rev. D 30 (1984) 130 [inSPIRE].

[50] H.H. Patel, Package-X: A Mathematica package for the analytic calculation of one-loop integrals, Comput. Phys. Commun. 197 (2015) 276 [arXiv:1503.01469] [INSPIRE].

[51] A.Y. Smirnov and X.-J. Xu, Neutrino mixing in $\mathrm{SO}(10)$ GUTs with a non-Abelian flavor symmetry in the hidden sector, Phys. Rev. D 97 (2018) 095030 [arXiv:1803.07933] [INSPIRE].

[52] W. Rodejohann and X.-J. Xu, Trimaximal $\mu-\tau$ reflection symmetry, Phys. Rev. D 96 (2017) 055039 [arXiv: 1705. 02027] [INSPIRE]. 
[53] W. Rodejohann and X.-J. Xu, A left-right symmetric flavor symmetry model, Eur. Phys. J. C 76 (2016) 138 [arXiv: 1509.03265] [INSPIRE].

[54] J.A. Casas and A. Ibarra, Oscillating neutrinos and $\mu \rightarrow e, \gamma$, Nucl. Phys. B 618 (2001) 171 [hep-ph/0103065] [INSPIRE].

[55] J.M. Pearson et al., Unified equations of state for cold non-accreting neutron stars with Brussels-Montreal functionals - I. Role of symmetry energy, Mon. Not. Roy. Astron. Soc. 481 (2018) 2994 [Erratum ibid. 486 (2019) 768] [arXiv: 1903.04981] [INSPIRE].

[56] T.A. Wagner, S. Schlamminger, J.H. Gundlach and E.G. Adelberger, Torsion-balance tests of the weak equivalence principle, Class. Quant. Grav. 29 (2012) 184002 [arXiv:1207.2442] [INSPIRE].

[57] E.G. Adelberger, J.H. Gundlach, B.R. Heckel, S. Hoedl and S. Schlamminger, Torsion balance experiments: A low-energy frontier of particle physics, Prog. Part. Nucl. Phys. 62 (2009) 102 [INSPIRE].

[58] E.G. Adelberger, B.R. Heckel, S.A. Hoedl, C.D. Hoyle, D.J. Kapner and A. Upadhye, Particle Physics Implications of a Recent Test of the Gravitational Inverse Sqaure Law, Phys. Rev. Lett. 98 (2007) 131104 [hep-ph/0611223] [INSPIRE].

[59] M. Bordag, U. Mohideen and V.M. Mostepanenko, New developments in the Casimir effect, Phys. Rept. 353 (2001) 1 [quant-ph/0106045] [INSPIRE].

[60] S. Davidson, S. Hannestad and G. Raffelt, Updated bounds on millicharged particles, JHEP 05 (2000) 003 [hep-ph/0001179] [INSPIRE].

[61] J. Redondo and G. Raffelt, Solar constraints on hidden photons re-visited, JCAP 08 (2013) 034 [arXiv: 1305.2920] [inSPIRE].

[62] C. Boehm, M.J. Dolan and C. McCabe, Increasing Neff with particles in thermal equilibrium with neutrinos, JCAP 12 (2012) 027 [arXiv: 1207.0497] [INSPIRE].

[63] A. Kamada and H.-B. Yu, Coherent Propagation of PeV Neutrinos and the Dip in the Neutrino Spectrum at IceCube, Phys. Rev. D 92 (2015) 113004 [arXiv:1504.00711] [INSPIRE].

[64] G.-y. Huang, T. Ohlsson and S. Zhou, Observational Constraints on Secret Neutrino Interactions from Big Bang Nucleosynthesis, Phys. Rev. D 97 (2018) 075009 [arXiv: 1712.04792] [INSPIRE].

[65] A. Kamada, K. Kaneta, K. Yanagi and H.-B. Yu, Self-interacting dark matter and muon $g-2$ in a gauged $\mathrm{U}(1)_{L_{\mu}-L_{\tau}}$ model, JHEP 06 (2018) 117 [arXiv: 1805.00651] [INSPIRE].

[66] X. Luo, W. Rodejohann and X.-J. Xu, Dirac neutrinos and $N_{\text {eff }}$, JCAP 06 (2020) 058 [arXiv: 2005.01629] [INSPIRE].

[67] K. Choi, C.W. Kim, J. Kim and W.P. Lam, Constraints on the Majoron Interactions From the Supernova SN1987A, Phys. Rev. D 37 (1988) 3225 [InSPIRE].

[68] K. Choi and A. Santamaria, Majorons and Supernova Cooling, Phys. Rev. D 42 (1990) 293 [INSPIRE].

[69] M. Kachelriess, R. Tomas and J.W.F. Valle, Supernova bounds on Majoron emitting decays of light neutrinos, Phys. Rev. D 62 (2000) 023004 [hep-ph/0001039] [INSPIRE].

[70] S. Hannestad, P. Keranen and F. Sannino, A Supernova constraint on bulk Majorons, Phys. Rev. D 66 (2002) 045002 [hep-ph/0204231] [INSPIRE]. 
[71] Y. Farzan, Bounds on the coupling of the Majoron to light neutrinos from supernova cooling, Phys. Rev. D 67 (2003) 073015 [hep-ph/0211375] [InSPIRE].

[72] J.B. Dent, F. Ferrer and L.M. Krauss, Constraints on Light Hidden Sector Gauge Bosons from Supernova Cooling, arXiv:1201.2683 [INSPIRE].

[73] H.K. Dreiner, J.-F. Fortin, C. Hanhart and L. Ubaldi, Supernova constraints on MeV dark sectors from $e^{+} e^{-}$annihilations, Phys. Rev. D 89 (2014) 105015 [arXiv:1310.3826] [INSPIRE].

[74] S. Bilmis, I. Turan, T.M. Aliev, M. Deniz, L. Singh and H.T. Wong, Constraints on Dark Photon from Neutrino-Electron Scattering Experiments, Phys. Rev. D 92 (2015) 033009 [arXiv: 1502.07763] [INSPIRE].

[75] M. Lindner, W. Rodejohann and X.-J. Xu, Coherent Neutrino-Nucleus Scattering and new Neutrino Interactions, JHEP 03 (2017) 097 [arXiv: 1612.04150] [INSPIRE].

[76] Y. Farzan, M. Lindner, W. Rodejohann and X.-J. Xu, Probing neutrino coupling to a light scalar with coherent neutrino scattering, JHEP 05 (2018) 066 [arXiv:1802.05171] [INSPIRE].

[77] M. Lindner, F.S. Queiroz, W. Rodejohann and X.-J. Xu, Neutrino-electron scattering: general constraints on $Z$ and dark photon models, JHEP 05 (2018) 098 [arXiv:1803.00060] [INSPIRE].

[78] A.N. Khan, W. Rodejohann and X.-J. Xu, Borexino and general neutrino interactions, Phys. Rev. D 101 (2020) 055047 [arXiv: 1906.12102] [inSPIRE].

[79] J.M. Link and X.-J. Xu, Searching for BSM neutrino interactions in dark matter detectors, JHEP 08 (2019) 004 [arXiv: 1903.09891] [INSPIRE].

[80] T. Kumar Poddar, S. Mohanty and S. Jana, Vector gauge boson radiation from compact binary systems in a gauged $L_{\mu}-L_{\tau}$ scenario, Phys. Rev. D 100 (2019) 123023 [arXiv: 1908.09732] [INSPIRE].

[81] D. Reitze et al., Cosmic Explorer: The U.S. Contribution to Gravitational-Wave Astronomy beyond LIGO, Bull. Am. Astron. Soc. 51 (2019) 035 [arXiv:1907.04833] [InSPIRE].

[82] S.L. Glashow, J. Iliopoulos and L. Maiani, Weak Interactions with Lepton-Hadron Symmetry, Phys. Rev. D 2 (1970) 1285 [INSPIRE]. 